\title{
Global Stability Analysis of Coupled-Oscillator Systems
}

\author{
Almudena Suárez, Fellow IEEE, Franco Ramírez, Sergio Sancho, Juan-Mari Collantes
}

\begin{abstract}
A methodology for the prediction and in-depth understanding of the stability properties of coupled-oscillator systems is presented. Unlike former investigations, all possible combinations of phase-shift values between the oscillator elements are considered. This provides greater insight into the mechanisms leading to instability. The analysis is based on the determination of the bifurcation loci in the space defined by the inter-stage phase shifts, enabling the detection of both ordinary and co-dimension two bifurcations. The new methodology for bifurcation detection is applicable to any number $\mathrm{N}$ of oscillator elements. For illustration, the case of three oscillator elements is considered. This is representative of the behavior for any number $\mathbf{N}$ and admits a planar representation of the bifurcation loci. The loci facilitate the comprehension of stability changes commonly observed during the system tuning and enable the evaluation and increase of the stability margins. Using these loci, it is possible to predict and synthesize the stable phase-shift regions, which will have interest in the case of non-constant distributions used in null formation and other applications. Good agreement has been found between simulations and measurements of a practical coupled-oscillator system at $3.85 \mathrm{GHz}$.
\end{abstract}

Index Terms-Coupled-oscillator system, stability analysis, bifurcation analysis.

\section{INTRODUCTION}

$\mathrm{C}$ OOUPLED-OSCILLATOR systems are applied for spatial power combining and beam steering of phased-array antennas [1]-[5]. As demonstrated in [1], [2], when the oscillators are synchronized, it is possible to obtain a constant phase shift progression between the oscillator elements by tuning the peripheral elements only. Non-constant phase-shift distributions can also be synthesized for null formation and other applications related to beam forming [6]-[9]. The coupled system often operates under weak coupling conditions, which reduces the amplitude and frequency variations when changing the inter-oscillator phase shift and virtually eliminates the risk of oscillation extinction in any of the oscillator elements [10]. Several works [1]-[4], [10]-[17] have addressed the stability analysis of coupled-oscillator systems with constant phase-shift distributions. Their main

Manuscript submitted February 10, 2014

A. Suárez, F. Ramírez and S. Sancho are with Dpto. Ingeniería de Comunicaciones, Universidad de Cantabria, Santander, 39005, Spain (e-mail: suareza@unican.es; ramirezf@unican.es; sanchosm@unican.es);

J-M Collantes is with the Dpto. de Electricidad y Electrónica, University of the Basque Country (UPV/EHU), Bilbao, 48080, Spain (e-mail: juanmari.collantes@ehu.es). goal has been to check the physical existence of particular solutions and/or determine the constant phase-shift interval with stable behavior. For a one-dimensional array of $\mathrm{N}$ oscillators one could expect, in principle, up to $2^{\mathrm{N}-1}$ coexisting solutions with different stability properties [3], [11]-[12], [14]. In [11], an effort was made to analyze all the solutions coexisting with the one that exhibits a constant phase progression. This was done for a standard design of the coupling network [1]-[3] with a one wavelength transmission line bounded by two resistors, in a series connection. However, to our knowledge, no previous work has addressed the stability analysis of all possible combinations of phaseshift values and the investigation of the bifurcations, or qualitative stability changes $[12,18]$, undergone by the coupled system when varying the tuning parameters. This analysis will provide valuable information on the various instability phenomena commonly observed during the system tuning. Furthermore, it will enable a global determination of all the stable (physical) phase-shift combinations in a given coupled-oscillator system. This information can be used at the design stage for an evaluation and increase of the stability margins. It will also be of interest in applications requiring non-constant phase-shift distributions.

The analysis will be based on the determination of the bifurcation loci in the space defined by the different interstage phase-shifts. As will be shown, using these analysis parameters, solutions that may coexist for the same values of the system parameters are univocally defined, which greatly facilitates the global determination of all the stable phase-shift combinations. The analysis is applicable to any number $\mathrm{N}$ of oscillator elements. For illustration, a "canonical" system of three oscillator elements will be considered here. In previous works [14]-[15], this system has been found to be representative of coupled systems with any number $\mathrm{N}$ of oscillator elements. With $N=3$ it will be possible to illustrate the various bifurcation phenomena [12], [18] through a planar tracing of two-dimensional bifurcation loci. To our knowledge, this is the first in-depth analysis of the bifurcation mechanisms delimiting the stable operation ranges of coupledoscillator systems. The analysis provides the D-type and Hopf bifurcation loci, as well as the co-dimension two bifurcations [18]-[19], requiring the fine tuning of two parameters. The double-zero eigenvalue bifurcations [18] (of co-dimension two) correspond to the distinct points at which the Hopf locus and the D-type locus merge. A simple analytical expression for the D-type bifurcation locus is derived, which is demonstrated to depend only on one angle, determined by the coupling network and the individual oscillator model. This 
fully new analytical expression (derived for the general case of arbitrary inter-stage phase shifts) is complemented with mathematical conditions for the double-zero eigenvalue bifurcations. Together with additional information on the magnitudes affecting the Hopf locus, this investigation of the bifurcation behavior provides the designer with great insight and control over the coupled-system performance. Major novelties with respect to previous works are the extension of the stability analysis to the case of non-constant inter-stage phase shifts, the new methodology for bifurcation analysis in terms of the phase shift between the oscillator element, and the detailed bifurcation analysis of the coupled system, including analytical expressions. The boundedness of the inter-stage phase shifts enables an efficient and exhaustive calculation of all the coexisting solutions and their stability properties.

The paper is organized as follows. Section II presents the formulation used for the stability analysis of the coupledoscillator system in the general case of non-constant interstage phase shift. Section III presents the Hopf and D-type bifurcation conditions, the analytical expressions for the Dtype locus and the mathematical conditions for the doublezero eigenvalue bifurcation. In section IV the mathematical expressions are applied to an array of three oscillators at $3.85 \mathrm{GHz}$, studying the evolution of bifurcation loci when varying the length of the transmission line in the coupling networks. Their impact on the location and size of the stable regions will be determined with the aid of these analyses. Stability of coexisting steady-state solutions is addressed in section $\mathrm{V}$.

\section{FORMULATION FOR THE STABILITY ANALYSIS WITH ARBITRARY PHASE-SHIFTS}

The formulation for the stability analysis of coupled oscillators in weak coupling conditions and with arbitrary phase-shifts will be developed for a three-oscillator system [Fig. 1(a)]. As demonstrated in the works [14]-[15], limited to the case of a constant phase-shift progression, under weakcoupling conditions, the steady-state response with any number $N$ of oscillator elements can be inferred from that of a system of three elements $N=3$. In the following stability analysis, all possible values of the two inter-oscillator phase shifts $\phi_{1,2}=\phi_{2}-\phi_{1}$ and $\phi_{2,3}=\phi_{3}-\phi_{2}$ will be considered. The oscillator elements are assumed equal. When isolated from the system, they oscillate at the free-running frequency $\omega_{0}$ and voltage amplitude $\mathrm{V}_{0}$ (at the connection node), for the tuning parameter value $\eta_{0}$. Under weak coupling conditions, the current to voltage ratio (admittance function Y) of each oscillator element will only undergo a small increment with respect to the free-running value $Y_{0}=0$. This is determined by its derivatives with respect to the voltage $\left(\mathrm{Y}_{\mathrm{V}}\right)$, frequency $\left(\mathrm{Y}_{\omega}\right)$, and tuning parameter $\left(\mathrm{Y}_{\eta}\right)$, evaluated at the free-running point. Applying Kirchoff's laws to the $N=3$ oscillator nodes at the fundamental frequency, one obtains the following system of steady-state equations $\mathrm{H}_{1}$ to $\mathrm{H}_{3}$ :

$$
\begin{aligned}
& \mathrm{H}_{1}=\mathrm{Y}_{\mathrm{V}} \mathrm{V}_{0} \Delta \mathrm{V}_{1}+\mathrm{Y}_{\omega} \mathrm{V}_{0} \Delta \omega+\mathrm{Y}_{\eta} \mathrm{V}_{0} \Delta \eta_{1}+\mathrm{Y}_{\mathrm{e}} \mathrm{V}_{0}+ \\
& +\mathrm{Y}_{\mathrm{nb}} \mathrm{V}_{0} \mathrm{e}^{\mathrm{j}\left(\phi_{2}-\phi_{1}\right)}=0 \\
& \mathrm{H}_{2}=\mathrm{Y}_{\mathrm{V}} \mathrm{V}_{0} \Delta \mathrm{V}_{2}+\mathrm{Y}_{\omega} \mathrm{V}_{0} \Delta \omega+\mathrm{Y}_{\mathrm{nb}} \mathrm{V}_{0} \mathrm{e}^{\mathrm{j}\left(\phi_{1}-\phi_{2}\right)}+ \\
& +2 \mathrm{Y}_{\mathrm{e}} \mathrm{V}_{0}+\mathrm{Y}_{\mathrm{nb}} \mathrm{V}_{0} \mathrm{e}^{\mathrm{j}\left(\phi_{3}-\phi_{2}\right)}=0 \\
& \mathrm{H}_{3}=\mathrm{Y}_{\mathrm{V}} \mathrm{V}_{0} \Delta \mathrm{V}_{3}+\mathrm{Y}_{\omega} \mathrm{V}_{0} \Delta \omega+\mathrm{Y}_{\eta} \mathrm{V}_{0} \Delta \eta_{2}+\mathrm{Y}_{\mathrm{nb}} \mathrm{V}_{0} \mathrm{e}^{\mathrm{j}\left(\phi_{2}-\phi_{3}\right)}+ \\
& +\mathrm{Y}_{\mathrm{e}} \mathrm{V}_{0}=0
\end{aligned}
$$

where $Y_{e}$ and $Y_{n b}$ correspond to the parameters $Y_{11}$ and $Y_{12}$ of the admittance matrix of the coupling network, assumed passive and symmetrical, and $\Delta \mathrm{V}_{1}, \Delta \mathrm{V}_{2}, \Delta \mathrm{V}_{3}, \Delta \omega, \Delta \eta_{1}, \Delta \eta_{2}$ are the increments in the oscillation amplitude, oscillation frequency and tuning parameters with respect to the freerunning values $\left(\mathrm{V}_{0}, \omega_{0}, \eta_{0}\right)$. The phases of the three oscillator elements are $\phi_{1}, \phi_{2}, \phi_{3}$, though for the steady-state calculation, one of the them can be arbitrarily made equal to zero [12], [18], due to the system autonomy $\left(\phi_{1}=0^{\circ}\right)$. Note that the second order terms: $\Delta \mathrm{V}_{\mathrm{i}}^{2}, \Delta \mathrm{V}_{\mathrm{i}} \Delta \omega, \Delta \mathrm{V}_{\mathrm{i}} \Delta \eta$ with $i=1$ to 3 , have been neglected in (1). The system still has a non-constant amplitude distribution, due to the terms $\Delta \mathrm{V}_{\mathrm{i}}$ (where $i=1$ to 3 ).

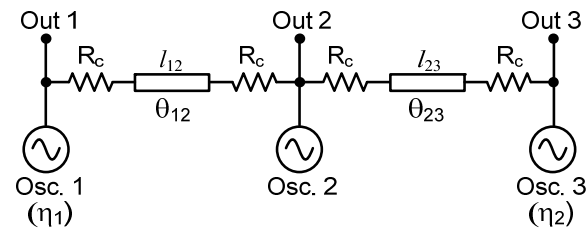

(a)

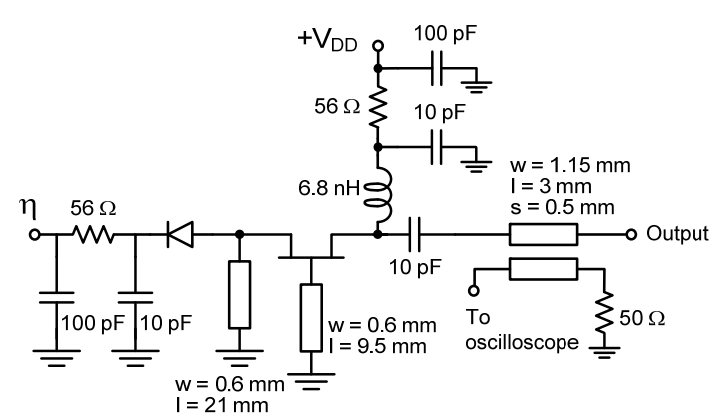

(b)

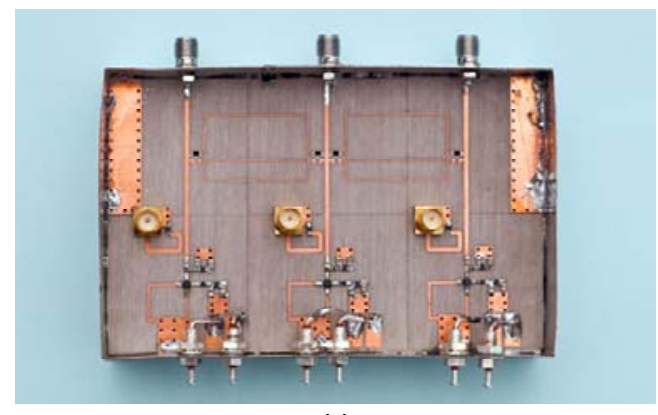

(c)

Fig. 1. Generic schematic of a three-element coupled-oscillator system. (a) The coupling network consists of a transmission line of length $l$ bounded by two resistors $R_{c}$ in series connection. (b) Schematic of the individual oscillator circuit based on the FET NE3210S01. (c) Photo of the three-element coupled-oscillator system. 
System (1) is linear in $\Delta \mathrm{V}_{\mathrm{i}}, \Delta \omega, \Delta \eta_{1}, \Delta \eta_{2}$ but nonlinear in the phase values. For a realistic description of the oscillator elements, the derivatives $Y_{V}, Y_{\omega}, Y_{\eta}$ are calculated through finite differences in harmonic balance (HB), using an auxiliary generator (AG), as shown in [20]. The inter-stage phase shifts $\phi_{1,2}=\phi_{2}-\phi_{1}=\phi_{2}$ and $\phi_{2,3}=\phi_{3}-\phi_{2}$ are modified by simultaneously varying the two independent tuning parameters $\Delta \eta_{1}, \Delta \eta_{2}$ of the two outermost oscillators. The formulation (1) is applicable to non-purely sinusoidal oscillators provided the harmonic content is relatively low, so that the currents injected by the coupling networks at higher harmonic components have a small effect on the coupled system behavior.

For the stability analysis, a small perturbation is considered in all the amplitude and phase variables of (1), which become:

$$
\begin{aligned}
& \mathrm{V}_{1}(\mathrm{t})=\mathrm{V}_{0}+\Delta \mathrm{V}_{1}+\delta \mathrm{V}_{1}(\mathrm{t}) \\
& \mathrm{V}_{2}(\mathrm{t})=\mathrm{V}_{0}+\Delta \mathrm{V}_{2}+\delta \mathrm{V}_{2}(\mathrm{t}) \\
& \mathrm{V}_{3}(\mathrm{t})=\mathrm{V}_{0}+\Delta \mathrm{V}_{3}+\delta \mathrm{V}_{3}(\mathrm{t}) \\
& \phi_{1}(\mathrm{t})=0+\delta \phi_{1}(\mathrm{t}) \\
& \phi_{2}(\mathrm{t})=\phi_{2}+\delta \phi_{2}(\mathrm{t}) \\
& \phi_{3}(\mathrm{t})=\phi_{3}+\delta \phi_{3}(\mathrm{t})
\end{aligned}
$$

where $\phi_{1}=0^{\circ}$ has been assumed, due to the system autonomy. Let the state-variable vector $\overline{\mathrm{X}}=\left(\Delta \mathrm{V}_{1}, \Delta \mathrm{V}_{2}, \Delta \mathrm{V}_{3}, 0, \phi_{2}, \phi_{3}\right)^{\mathrm{t}}$ be defined. Because the perturbation is small, it is possible to linearize the coupled system (1) about the steady-state solution $\overline{\mathrm{X}}_{\mathrm{o}}$. The linearization will also be carried out in terms of the frequency variable $\omega$, which is justified from the identical design of all the oscillator elements and the fact that they should be stable prior to their connection to the coupled system. In these conditions, instability can only arise as a result of the weak coupling and may only lead to unstable poles with zero or small imaginary part [1]-[3],[12]. This will be in the order of the difference between the individual oscillation frequencies. Performing this linearization, one obtains the following perturbed system:

$$
\begin{aligned}
& {\left[\mathrm{Y}_{\mathrm{V}}\left(\Delta \mathrm{V}_{1}+\delta \mathrm{V}_{1}(\mathrm{t})\right)+\mathrm{Y}_{\omega}(\Delta \omega+\mathrm{s} / \mathrm{j})+\mathrm{Y}_{\eta} \Delta \mathrm{\eta}_{1}\right] \mathrm{V}_{1}(\mathrm{t}) \mathrm{e}^{\mathrm{j} \phi_{1}(\mathrm{t})}=} \\
& =-\mathrm{Y}_{\mathrm{e}} \mathrm{V}_{0} \mathrm{e}^{\mathrm{j} \phi_{1}(\mathrm{t})}-\mathrm{Y}_{\mathrm{nb}} \mathrm{V}_{0} \mathrm{e}^{\mathrm{j} \phi_{2}(\mathrm{t})} \\
& {\left[\mathrm{Y}_{\mathrm{V}}\left(\Delta \mathrm{V}_{2}+\delta \mathrm{V}_{2}(\mathrm{t})\right)+\mathrm{Y}_{\omega}(\Delta \omega+\mathrm{s} / \mathrm{j})\right] \mathrm{V}_{2}(\mathrm{t}) \mathrm{e}^{\mathrm{j} \phi_{2}(\mathrm{t})}=} \\
& =-\mathrm{Y}_{\mathrm{nb}} \mathrm{V}_{0} \mathrm{e}^{\mathrm{j} \phi_{1}(\mathrm{t})}-2 \mathrm{Y}_{\mathrm{e}} \mathrm{V}_{0} \mathrm{e}^{\mathrm{j} \phi_{2}(\mathrm{t})}-\mathrm{Y}_{\mathrm{nb}} \mathrm{V}_{0} \mathrm{e}^{\mathrm{j} \phi_{3}(\mathrm{t})} \\
& {\left[\mathrm{Y}_{\mathrm{V}}\left(\Delta \mathrm{V}_{3}+\delta \mathrm{V}_{3}(\mathrm{t})\right)+\mathrm{Y}_{\omega}(\Delta \omega+\mathrm{s} / \mathrm{j})+\mathrm{Y}_{\eta} \Delta \eta_{2}\right] \mathrm{V}_{3}(\mathrm{t}) \mathrm{e}^{\mathrm{j} \phi_{3}(\mathrm{t})}=} \\
& =-\mathrm{Y}_{\mathrm{nb}} \mathrm{V}_{0} \mathrm{e}^{\mathrm{j} \phi_{2}(\mathrm{t})}-\mathrm{Y}_{\mathrm{e}} \mathrm{V}_{0} \mathrm{e}^{\mathrm{j} \phi_{3}(\mathrm{t})}
\end{aligned}
$$

where $s$ is the derivative operator. The exponentials of the phase variables can be approached as:

$$
\mathrm{e}^{\mathrm{j} \phi_{\mathrm{i}}(\mathrm{t})} \square \mathrm{e}^{\mathrm{j} \phi_{\mathrm{i}}}\left(1+\mathrm{j} \delta \phi_{\mathrm{i}}(\mathrm{t})\right)
$$

where $i=1$ to 3 . Application of the derivative operator $s$ to any of the time varying terms $V_{i}(t) e^{j_{i}(t)}$ provides the following increments:

$$
\left(\frac{\delta \dot{V}_{i}(t)}{\mathrm{V}_{0}}+j \delta \dot{\phi}_{\mathrm{i}}(\mathrm{t})\right) \mathrm{V}_{0} \mathrm{e}^{\mathrm{j} \phi_{\mathrm{i}}}
$$

where the dot indicates a time derivation and the second-order terms have been neglected. Replacing (5) and (4) into (3), and retaining only the first order terms [in consistency with system (1)], one obtains the following differential equation system:

$$
\begin{aligned}
& Y_{V} \Delta V_{1} V_{0} e^{j \phi_{1}}+Y_{V} \delta V_{1}(t) V_{0} e^{j \phi_{1}}+Y_{\omega} \Delta \omega V_{0} e^{j \phi_{1}}+ \\
& +Y_{\omega}\left(\delta \dot{\phi}_{1}-j \frac{\delta \dot{V}_{1}}{V_{0}}\right) V_{0} e^{j \phi_{i}}+ \\
& +Y_{\eta} \Delta \eta_{1} V_{0} e^{j \phi_{1}}+Y_{e} V_{0} e^{j \phi_{1}}+Y_{e} V_{0} j \delta \phi_{1}(t) e^{j \phi_{1}}+ \\
& +Y_{n b} V_{0} e^{j \phi_{2}}+Y_{n b} V_{0} j \delta \phi_{2}(t) e^{j \phi_{2}}=0 \\
& Y_{V} \Delta V_{2} V_{0} e^{j \phi_{2}}+Y_{V} \delta V_{2}(t) V_{0} e^{j \phi_{2}}+Y_{\omega} \Delta \omega V_{0} e^{j \phi_{2}}+ \\
& +Y_{\omega}\left(\delta \dot{\phi}_{2}-j \frac{\delta V_{2}}{V_{0}}\right) V_{0} e^{j \phi_{2}}+ \\
& +Y_{n b} V_{0} e^{j \phi_{1}}+Y_{n b} V_{0} j \delta \phi_{1}(t) e^{j \phi_{1}}+2 Y_{e} V_{0} e^{j \phi_{2}}+ \\
& +2 Y_{e} V_{0} j \delta \phi_{2}(t) e^{j \phi_{2}}+ \\
& +Y_{n b} V_{0} e^{j \phi_{3}}+Y_{n b} V_{0} j \delta \phi_{3}(t) e^{j \phi_{3}}=0 \\
& Y_{V} \Delta V_{3} V_{0} e^{j \phi_{3}}+Y_{V} \delta V_{3}(t) V_{0} e^{j \phi_{3}}+Y_{\omega} \Delta \omega V_{0} e^{j \phi_{3}}+ \\
& +Y_{\omega}\left(\delta \dot{\phi}_{3}-j \frac{\delta \dot{V}_{3}}{V_{0}}\right) V_{0} e^{j \phi_{3}}+ \\
& +Y_{\eta} \Delta \eta_{2} V_{0} e^{j \phi_{3}}+Y_{n b} V_{0} e^{j \phi_{2}}+Y_{n b} V_{0} j \delta \phi_{2}(t) e^{j \phi_{2}}+ \\
& +Y_{e} V_{0} e^{j \phi_{3}}+Y_{e} V_{0} j \delta \phi_{3}(t) e^{j \phi_{3}}=0
\end{aligned}
$$

To obtain a compact equation in matrix form, the following vector will be defined:

$$
\overline{\mathrm{H}}=\left(\mathrm{H}_{1}^{\mathrm{r}}, \mathrm{H}_{1}^{\mathrm{i}}, \mathrm{H}_{2}^{\mathrm{r}}, \mathrm{H}_{2}^{\mathrm{i}}, \mathrm{H}_{3}^{\mathrm{r}}, \mathrm{H}_{3}^{\mathrm{i}}\right)^{\mathrm{T}}
$$

where the sub-indexes in $\overline{\mathrm{H}}$ refer to the components of the steady-state system in (1) and the super-indexes indicate real and imaginary parts. After cancelling the steady-state terms in (6), the following Linear Time Invariant (LTI) system in the time-varying increments is obtained:

$$
\delta \dot{\overline{\mathrm{X}}}(\mathrm{t})=\left[\begin{array}{c}
\delta \dot{\mathrm{V}}_{1}(\mathrm{t}) \\
\delta \dot{\mathrm{V}}_{2}(\mathrm{t}) \\
\delta \dot{\mathrm{V}}_{3}(\mathrm{t}) \\
\delta \dot{\phi}_{1}(\mathrm{t}) \\
\delta \dot{\phi}_{2}(\mathrm{t}) \\
\delta \dot{\phi}_{3}(\mathrm{t})
\end{array}\right]=\left.\left.\frac{\partial \overline{\mathrm{H}}}{\partial \dot{\overline{\mathrm{X}}}}\right|_{\overline{\mathrm{X}}_{0}} ^{-1} \cdot \frac{\partial \overline{\mathrm{H}}}{\partial \overline{\mathrm{X}}}\right|_{\overline{\mathrm{X}}_{0}}\left[\begin{array}{c}
\delta \mathrm{V}_{1}(\mathrm{t}) \\
\delta \mathrm{V}_{2}(\mathrm{t}) \\
\delta \mathrm{V}_{3}(\mathrm{t}) \\
\delta \phi_{1}(\mathrm{t}) \\
\delta \phi_{2}(\mathrm{t}) \\
\delta \phi_{3}(\mathrm{t})
\end{array}\right]=[\mathrm{M}] \delta \overline{\mathrm{X}}(\mathrm{t})
$$

where $\bar{X}_{o}$ is the steady-state solution of (1), at which the derivatives in (6) are calculated and the vector $\delta \bar{X}(t)$ of timevarying increments has been defined. The derivative matrixes 
in (8), obtained from simple inspection of system (6), are given by:

$$
\begin{aligned}
& \left.\frac{\partial \overline{\mathrm{H}}}{\partial \dot{\overline{\mathrm{X}}}}\right|_{\overline{\mathrm{X}}_{0}}=\left[\begin{array}{cccccc}
\frac{\mathrm{Y}_{\omega}^{\mathrm{i}}}{\mathrm{V}_{0}} & 0 & 0 & \mathrm{Y}_{\omega}^{\mathrm{r}} & 0 & 0 \\
-\frac{\mathrm{Y}_{\omega}^{\mathrm{r}}}{\mathrm{V}_{0}} & 0 & 0 & \mathrm{Y}_{\omega}^{\mathrm{i}} & 0 & 0 \\
0 & \frac{\mathrm{Y}_{\omega}^{\mathrm{i}}}{\mathrm{V}_{0}} & 0 & 0 & \mathrm{Y}_{\omega}^{\mathrm{r}} & 0 \\
0 & -\frac{\mathrm{Y}_{\omega}^{\mathrm{r}}}{\mathrm{V}_{0}} & 0 & 0 & \mathrm{Y}_{\omega}^{\mathrm{i}} & 0 \\
0 & 0 & \frac{\mathrm{Y}_{\omega}^{\mathrm{i}}}{\mathrm{V}_{0}} & 0 & 0 & \mathrm{Y}_{\omega}^{\mathrm{r}} \\
0 & 0 & -\frac{\mathrm{Y}_{\omega}^{\mathrm{r}}}{\mathrm{V}_{0}} & 0 & 0 & \mathrm{Y}_{\omega}^{\mathrm{i}}
\end{array}\right] \\
& \left.\frac{\partial \overline{\mathrm{H}}}{\partial \overline{\mathrm{X}}}\right|_{\overline{\mathrm{X}}_{0}}=\left[\begin{array}{cccccc}
-\mathrm{Y}_{\mathrm{v}}^{\mathrm{r}} & 0 & 0 & \frac{\partial \mathrm{a}^{\mathrm{r}}}{\partial \phi_{1}} & \frac{\partial \mathrm{a}^{\mathrm{r}}}{\partial \phi_{2}} & 0 \\
-\mathrm{Y}_{\mathrm{v}}^{\mathrm{i}} & 0 & 0 & \frac{\partial \mathrm{a}^{\mathrm{i}}}{\partial \phi_{1}} & \frac{\partial \mathrm{a}^{\mathrm{i}}}{\partial \phi_{2}} & 0 \\
0 & -\mathrm{Y}_{\mathrm{v}}^{\mathrm{r}} & 0 & \frac{\partial \mathrm{b}^{\mathrm{r}}}{\partial \phi_{1}} & \frac{\partial \mathrm{b}^{\mathrm{r}}}{\partial \phi_{2}} & \frac{\partial \mathrm{b}^{\mathrm{r}}}{\partial \phi_{3}} \\
0 & -Y_{\mathrm{v}}^{\mathrm{i}} & 0 & \frac{\partial \mathrm{b}^{\mathrm{i}}}{\partial \phi_{1}} & \frac{\partial \mathrm{b}^{\mathrm{i}}}{\partial \phi_{2}} & \frac{\partial \mathrm{b}^{\mathrm{i}}}{\partial \phi_{3}} \\
0 & 0 & -\mathrm{Y}_{\mathrm{v}}^{\mathrm{r}} & 0 & \frac{\partial \mathrm{c}^{\mathrm{r}}}{\partial \phi_{2}} & \frac{\partial \mathrm{c}^{\mathrm{r}}}{\partial \phi_{3}} \\
0 & 0 & -\mathrm{Y}_{\mathrm{v}}^{\mathrm{i}} & 0 & \frac{\partial \mathrm{c}^{\mathrm{i}}}{\partial \phi_{2}} & \frac{\partial \mathrm{c}^{\mathrm{i}}}{\partial \phi_{3}}
\end{array}\right]
\end{aligned}
$$

Note that the functions $a^{\mathrm{r}}, \mathrm{a}^{\mathrm{i}}, \mathrm{b}^{\mathrm{r}}, \mathrm{b}^{\mathrm{i}}, \mathrm{c}^{\mathrm{r}}, \mathrm{c}^{\mathrm{i}}$ in the above matrix correspond to the real and imaginary parts of the phase dependent terms in (1), that is:

$$
\begin{aligned}
& a^{r}=-Y_{e}^{r}-Y_{n b}^{r} \cos \left(\phi_{2}-\phi_{1}\right)+Y_{n b}^{i} \sin \left(\phi_{2}-\phi_{1}\right) \\
& a^{i}=-Y_{e}^{i}-Y_{n b}^{r} \sin \left(\phi_{2}-\phi_{1}\right)-Y_{n b}^{i} \cos \left(\phi_{2}-\phi_{1}\right) \\
& b^{\mathrm{r}}=-\mathrm{Y}_{\mathrm{nb}}^{\mathrm{r}} \cos \left(\phi_{2}-\phi_{1}\right)-\mathrm{Y}_{\mathrm{nb}}^{\mathrm{i}} \sin \left(\phi_{2}-\phi_{1}\right)-2 \mathrm{Y}_{\mathrm{e}}^{\mathrm{r}}- \\
& -Y_{\mathrm{nb}}^{\mathrm{r}} \cos \left(\phi_{3}-\phi_{2}\right)+\mathrm{Y}_{\mathrm{nb}}^{\mathrm{i}} \sin \left(\phi_{3}-\phi_{2}\right) \\
& b^{\mathrm{i}}=-Y_{\mathrm{nb}}^{\mathrm{i}} \cos \left(\phi_{2}-\phi_{1}\right)+Y_{\mathrm{nb}}^{\mathrm{r}} \sin \left(\phi_{2}-\phi_{1}\right)-2 Y_{\mathrm{e}}^{\mathrm{i}}- \\
& \text { - } \mathrm{Y}_{\mathrm{nb}}^{\mathrm{r}} \sin \left(\phi_{3}-\phi_{2}\right)-\mathrm{Y}_{\mathrm{nb}}^{\mathrm{i}} \cos \left(\phi_{3}-\phi_{2}\right) \\
& c^{r}=-Y_{n b}^{r} \cos \left(\phi_{3}-\phi_{2}\right)-Y_{n b}^{i} \sin \left(\phi_{3}-\phi_{2}\right)-Y_{e}^{r} \\
& c^{\mathrm{i}}=-Y_{\mathrm{nb}}^{\mathrm{i}} \cos \left(\phi_{3}-\phi_{2}\right)+Y_{\mathrm{nb}}^{\mathrm{r}} \sin \left(\phi_{3}-\phi_{2}\right)-Y_{\mathrm{e}}^{\mathrm{i}}
\end{aligned}
$$

The stability of a particular steady-state solution $\overline{\mathrm{X}}_{\mathrm{o}}=\left(\Delta \mathrm{V}_{1}, \Delta \mathrm{V}_{2}, \Delta \mathrm{V}_{3}, 0, \phi_{1,2}, \phi_{2,3}\right)^{\mathrm{t}}$ is determined by the eigenvalues of the constant matrix $[\mathrm{M}]$ in (8). In the case considered here, $N=3$, the order of matrix [M] is $6 \times 6$. Note that when doing $\phi_{1,2}=\phi_{2,3}=\phi$, the formulation particularizes to the one in [14], which only considers the case of a constant inter-stage phase shift. Due to the autonomy of the system (1), the matrix $[\mathrm{M}]$ will have a zero eigenvalue for all the possible steady state solutions $\overline{\mathrm{X}}$, obtained by varying the tuning elements $\Delta \eta_{1}, \Delta \eta_{2}$. Therefore, the stability will be determined by the 5 remaining eigenvalues of $[\mathrm{M}]$, which can be real or complex conjugate.

\section{BIFURCATION ANALYSIS}

A key aspect to understand the stability properties of the coupled system is the fact that its eigenvalues vary in a continuous manner versus any analysis parameter. This comes from the continuity of the system equations. As gathered from inspection of (8), the functions on the right hand side of this equation system are derivable, so the system is continuous. The continuity of the eigenvalues implies that small changes in the operation conditions turn into small variations of the eigenvalues. Prior to the connection to the coupled system, each individual oscillator in free-running conditions contains a zero eigenvalue, associated to solution autonomy. After its introduction into a weakly-coupled system, these critical eigenvalues will undergo a small variation, except one of them $\left(\lambda_{1}=0\right)$, which will necessary remain at zero due to the autonomous behavior of the coupled system. Therefore, in weak coupling conditions an $\mathrm{N}$-oscillator system will have $\mathrm{N}-1$ eigenvalues located relatively close to the imaginary axis. For stability, these $N-1$ eigenvalues must be on the left-hand side of the complex plane.

Departing from the formulation developed in the previous section, in Sub-Section III.A we will present mathematical conditions enabling the detection of Hopf and D-type bifurcations, which, respectively, correspond to the crossing of a pair of complex-conjugate eigenvalues and a real eigenvalue through the imaginary axis [12], [18]-[19],[21]. A major advantage of the new method comes from the fact that detection of bifurcation points does not require obtaining the steady state solution in (1). Bifurcations can be detected from the analysis of Jacobian matrixes depending only on the individual oscillator derivatives $Y_{V}, Y_{\omega}, Y_{\eta}$, the admittance matrix of the coupling network and the particular phase shift values $\phi_{1,2}$ and $\phi_{2,3}$. In Sub-Section III.B an analytical expression for the D-type bifurcation locus will be presented, as well as the mathematical conditions for the occurrence of double-zero eigenvalue bifurcations (of co-dimension two), at which the Hopf locus and the D-type locus merge [18].

\section{A. Calculation of bifurcation loci}

The poles associated to a given steady-state solution will correspond to the roots of the characteristic determinant [12], [18]-[19] of the Linear Time Invariant (LTI) system (8), expressed in terms of the complex frequency $s$ :

$$
\begin{aligned}
& \operatorname{det}(\mathrm{s}[\mathrm{I}]-[\mathrm{M}])=\mathrm{p}_{6} \mathrm{~s}^{6}+\mathrm{p}_{5} \mathrm{~s}^{5}+\ldots+\mathrm{p}_{2} \mathrm{~s}^{2}+\mathrm{p}_{1} \mathrm{~s}= \\
& =\mathrm{s}\left(\mathrm{p}_{6} \mathrm{~s}^{5}+\mathrm{p}_{5} \mathrm{~s}^{4}+\ldots+\mathrm{p}_{2} \mathrm{~s}+\mathrm{p}_{1}\right)=0
\end{aligned}
$$

where $[\mathrm{I}]$ is the $6 \times 6$ identity matrix and the coefficients $\mathrm{p}_{\mathrm{i}}$, with $i=1$ to 6 , depend on the individual oscillator derivatives $Y_{V}, Y_{\omega}$, the coupling parameter $Y_{n b}$, the free- 
running oscillation amplitude $\mathrm{V}_{0}$ and the phase shift values $\phi_{1,2}, \phi_{2,3}$. Equation (12) has a root $s=0$ due to the autonomous behavior of the coupled-oscillator system. Dividing the polynomial expression by $s$ and replacing $s$ with the imaginary term $j \Omega$, we will have the following bifurcation condition:

$$
p_{6}(j \Omega)^{5}+p_{5}(j \Omega)^{4}+\ldots+p_{2}(j \Omega)+p_{1}=0
$$

Hopf bifurcations are obtained for $\mathrm{j} \Omega \neq 0$ and D-type bifurcations are obtained for $j \Omega=0$. In the following, a detailed description of the implications of these two bifurcations and the methodologies for their practical numerical/analytical calculation will be presented. The relevant case of double-zero eigenvalues, of co-dimension two, will also be considered.

\section{1) Hopf bifurcation}

Let the fundamental frequency of the periodic solution of the coupled oscillator be $\omega$. At a Hopf bifurcation [12], [18][19], [21] a pair of complex-conjugate eigenvalues $\sigma \pm \mathrm{j} \Omega$, where $\Omega \neq 0$ is incommensurable with $\omega$, crosses the imaginary axis of the complex plane. Incommensurable frequencies are not harmonically related, which implies $\Omega \neq \frac{\mathrm{m}}{\mathrm{n}} \omega$, where $m$ and $n$ are integers. The Hopf bifurcation gives rise to the onset of a quasi-periodic solution at the two fundamental frequencies $\omega, \Omega$. For the Hopf bifurcation detection, the complex equation (13) should be numerically solved in terms of $\phi_{1,2}, \phi_{2,3}$ and $\Omega$ through an error minimization technique. An alternative procedure, based on eigenvalue computation, is used in this work, noting that a Hopf bifurcation will occur at the pairs of inter-stage phase shift values $\phi_{1,2}, \phi_{2,3}$ (acting as the analysis parameters) that fulfill the condition:

$$
\sigma\left(\phi_{1,2}, \phi_{2,3}\right)=0, \quad \Omega\left(\phi_{1,2}, \phi_{2,3}\right) \neq 0
$$

The eigenvalues are calculated using LAPACK [22] routines to implement the associated matrix factorizations such as LU (Lower Upper), QR, Cholesky and Schur decomposition. The bifurcation detection will be based on the computation, classification and ordering of the eigenvalues of the matrix [M]. For each pair of inter-stage phase shift values $\phi_{1,2}, \phi_{2,3}$, the eigenvalues $\lambda_{\mathrm{i}}$ of the matrix $\left[\mathrm{M}\left(\phi_{1,2}, \phi_{2,3}\right)\right]$ are calculated and rearranged as:

$$
\left|\lambda_{1}^{r}\right| \leq\left|\lambda_{2}^{r}\right| \leq\left|\lambda_{3}^{r}\right| \leq \cdots \leq\left|\lambda_{2 N}^{r}\right|
$$

where the superindex $r$ means real part. The first eigenvalue $\lambda_{1}=0$ is associated to the system autonomy. Due to the arrangement in (15), if the third eigenvalue has a zero real part $\lambda_{3}^{r}=0$, the second eigenvalue will necessarily have a zero real part too $\lambda_{2}^{r}=0$. There are two possible causes for this situation. The first and more general is the Hopf bifurcation, for which two complex conjugate eigenvalues are located on the imaginary axis $\pm j \Omega$. The second one is the codimension-two bifurcation, corresponding to a degenerate Hopf bifurcation, for which the imaginary part of the complex-conjugate eigenvalues is equal to zero (double-zero eigenvalue bifurcation [18]). As will be shown in Sub-Section III.B, the co-dimension-two bifurcations correspond to distinct (isolated) points in the parameter plane. Note that at ordinary D-type bifurcations (where a single real eigenvalue crosses the imaginary axis) condition $\lambda_{3}^{r} \neq 0$ is fulfilled, due to the arrangement in (15).

Taking the above reasoning into account, Hopf bifurcations can be detected from the analysis of the real part of the third eigenvalue. For this detection, the following two-dimensional function will be defined:

$$
\sigma_{3}\left(\phi_{1,2}, \phi_{2,3}\right)=\lambda_{3}^{r}
$$

The function (16) provides a surface in the space $\phi_{1,2}, \phi_{2,3}$, which is obtained by two undemanding nested phase sweeps in $\phi_{1,2}, \phi_{2,3}$, bounded in the interval $\left(-180^{\circ}, 180^{\circ}\right)$. Then, the entire Hopf locus can be directly traced by obtaining the intersection of the surface (16) with the plane $\sigma_{3}=0$. Once the phase-shift values at the Hopf bifurcation locus have been calculated, the corresponding values of the tuning voltages can be directly obtained by solving the linear system (1).

\section{2) D-type bifurcation}

As already indicated, system (8) will exhibit an eigenvalue $\lambda_{1}=0$ for all the possible values of $\phi_{1,2}, \phi_{2,3}$, which is due to the system autonomy. The crossing of an additional real eigenvalue through zero $\left(\lambda_{2}=0\right)$ will give rise to a D-type bifurcation, associated with a singularity of the solution curve when traced versus any analysis parameter. In fact, D-type bifurcations, leading to qualitative stability changes at the fundamental frequency (without generation of any new frequency components in the solution spectrum) necessarily have an impact on the geometry of the solution curve [12], or set of solutions obtained when varying the analysis parameter. Note that any singularity of the matrix $[\mathrm{M}]$ in (8) will be due to a singularity of $[\mathrm{JX}]=\left.\frac{\partial \overline{\mathrm{H}}}{\partial \overline{\mathrm{X}}}\right|_{\overline{\mathrm{X}}_{0}}$ since the matrix $\left.\frac{\partial \overline{\mathrm{H}}}{\partial \dot{\overline{\mathrm{X}}}}\right|_{\overline{\mathrm{X}}_{0}} ^{-1}$ is constant for all the parameter values and nonsingular, as can be easily verified. Due to the free-running behavior of the coupled system, at the D-type bifurcation the Jacobian matrix [JX] will have two zero eigenvalues: one inherent to the system autonomy and the other due to the D-type bifurcation. Applying the properties of the square matrixes, the rank and Kernel dimension of $[\mathrm{JX}]$, denoted, respectively $\mathrm{R}_{\mathrm{X}}$ and $\mathrm{K}_{\mathrm{X}}$, will fulfill:

$$
\mathrm{K}_{\mathrm{x}}=6-\mathrm{R}_{\mathrm{x}}, \quad \mathrm{K}_{\mathrm{x}} \geq 1
$$

Note that the kernel dimension agrees with the number of zero eigenvalues. The condition $\mathrm{K}_{\mathrm{X}} \geq 1$ is due to the system autonomy. A new matrix [JC] will be defined here, which is obtained by replacing the column $\frac{\partial \overline{\mathrm{H}}}{\partial \phi_{1}}$ with $\frac{\partial \overline{\mathrm{H}}}{\partial \omega}$ in the matrix [JX], where $\omega$ is the angular frequency. Thus, the matrix [JC] 
can be expressed as:

$$
[\mathrm{JC}]=\left(\frac{\partial \overline{\mathrm{H}}}{\partial \mathrm{V}_{1}}, \frac{\partial \overline{\mathrm{H}}}{\partial \mathrm{V}_{2}}, \frac{\partial \overline{\mathrm{H}}}{\partial \mathrm{V}_{3}}, \frac{\partial \overline{\mathrm{H}}}{\partial \omega}, \frac{\partial \overline{\mathrm{H}}}{\partial \phi_{2}}, \frac{\partial \overline{\mathrm{H}}}{\partial \phi_{3}}\right)
$$

Since $[\mathrm{JX}]$ and $[\mathrm{JC}]$ differ only in one column, their rank can differ at maximum in one unity and, from equation (17):

$$
\left|\mathrm{K}_{\mathrm{JC}}-\mathrm{K}_{\mathrm{X}}\right| \leq 1
$$

Note that, any one of the last three columns of [JX] corresponding to the phase derivatives can be expressed as a linear combination of the other two. Since $\omega$ is an independent parameter, not expressible as a linear combination of the state variables $\left(\mathrm{V}_{1}, \mathrm{~V}_{2}, \mathrm{~V}_{3}, \phi_{2}, \phi_{3}\right)$, the replacement of $\frac{\partial \overline{\mathrm{H}}}{\partial \phi_{1}}$ by $\frac{\partial \overline{\mathrm{H}}}{\partial \omega}$ implies that $\mathrm{K}_{\mathrm{JC}}=\mathrm{K}_{\mathrm{JX}}-1$.

To assess the rank of [JC] we will take into account that steady-state system (1) can be solved in terms of $\overline{\mathrm{X}}_{\omega}=\left[\Delta \mathrm{V}_{1}, \Delta \mathrm{V}_{2}, \Delta \mathrm{V}_{3}, \Delta \omega, \phi_{2}, \phi_{3}\right]^{\mathrm{t}}$ for most pairs of tuningparameter values $\left(\Delta \eta_{1}, \Delta \eta_{2}\right)$. According to the Implicit Function Theorem [23], this implies that the Jacobian matrix [JC] is invertible at those parameter values, fulfilling $\mathrm{K}_{\mathrm{JC}}\left(\Delta \eta_{1}, \Delta \eta_{2}\right)=0$. From the matrix comparison above, matrix [JC] will be singular (and therefore non invertible) at the points $\left(\Delta \eta_{10}, \Delta \eta_{20}\right)$ at which $[\mathrm{JX}]$ exhibits two zero eigenvalues. Therefore, a singularity of matrix $[\mathrm{JC}]$ implies a D-type bifurcation.

The geometrical implications are demonstrated next. Let $\overline{\mathrm{X}}_{\omega}^{\mathrm{n}}$ be the point $\mathrm{n}$ of the solution curve obtained when varying any of the two tuning parameters $\Delta \eta_{\mathrm{i}}$, where $i=1,2$. Next point $n+1$, expressed as $\overline{\mathrm{X}}_{\omega}^{\mathrm{n}+1}$, and obtained after an arbitrarily small incremental change in the analysis parameter (either $\Delta \eta_{1}$ or $\left.\Delta \eta_{2}\right)$, can be approached:

$$
[\mathrm{JC}]\left(\begin{array}{llllll}
\delta \mathrm{V}_{1} & \delta \mathrm{V}_{2} & \delta \mathrm{V}_{3} & \delta \omega & \delta \phi_{2} & \delta \phi_{3}
\end{array}\right)^{\mathrm{T}}+\frac{\partial \overline{\mathrm{H}}}{\partial \eta_{\mathrm{i}}} \delta \eta_{\mathrm{i}}=0
$$

The above constant increments should not be confused with the time varying increments resulting from the system perturbation in (2). For compactness, the following vector will be defined:

$$
\delta \overline{\mathrm{X}}_{\omega}=\left(\delta \mathrm{V}_{1} \delta \mathrm{V}_{2} \delta \mathrm{V}_{3} \delta \omega \delta \phi_{2} \delta \phi_{3}\right)^{\mathrm{T}}
$$

Dividing both terms by the scalar $\delta \eta_{\mathrm{i}}$ and solving for the resulting vector one obtains:

$$
\lim _{\delta \eta_{\mathrm{i}} \rightarrow 0} \frac{\delta \overline{\mathrm{X}}_{\omega}}{\delta \eta_{\mathrm{i}}}=\frac{\mathrm{d} \overline{\mathrm{X}}_{\omega}}{\mathrm{d} \eta_{\mathrm{i}}}=-[\mathrm{JC}]^{-1} \frac{\partial \overline{\mathrm{H}}}{\partial \eta_{\mathrm{i}}}
$$

Therefore, a singular matrix [JC] implies an infinite slope of the solution curve (traced in terms of any of the variables $\left.\Delta \mathrm{V}_{1}, \Delta \mathrm{V}_{2}, \Delta \mathrm{V}_{3}, \Delta \omega, \phi_{2}, \phi_{3}\right)$ versus any of the two parameters $\eta_{\mathrm{i}}$, where $i=1,2$.

\section{B. Analytical expression of the D-type bifurcation}

Due to the existence of numerous zeroes in the matrix [JC], most coming from the three first columns of amplitude derivatives in (18), it will be possible to obtain a manageable analytical expression for the $\operatorname{determinant} \operatorname{det}[\mathrm{JC}]$. This determinant is operated first. Then, the various inner products and cross products existing in the resulting expression are carefully identified, which provides:

$$
\begin{aligned}
& \operatorname{det}[\mathrm{JC}]=\mathrm{C}\left[-\sin \left(\phi_{2,3}-\phi_{1,2}\right) \sin \alpha_{\mathrm{v}, \mathrm{nb}} \cos \alpha_{\mathrm{v}, \mathrm{nb}}-\right. \\
& -\frac{1}{2} \cos \left(\phi_{2,3}+\phi_{1,2}\right)\left(3-4 \sin ^{2} \alpha_{\mathrm{v}, \mathrm{nb}}\right)- \\
& \left.-\frac{1}{2} \cos \left(\phi_{2,3}-\phi_{1,2}\right)\left(1+2 \cos ^{2} \alpha_{\mathrm{v}, \mathrm{nb}}\right)\right]
\end{aligned}
$$

where the constant $\mathrm{C}$ is:

$$
\mathrm{C}=\left(\mathrm{Y}_{\mathrm{v}} \times \mathrm{Y}_{\omega}\right)\left|\mathrm{Y}_{\mathrm{v}}\right|^{2}\left|\mathrm{Y}_{\mathrm{nb}}\right|^{2}
$$

and $Y_{v} \times Y_{\omega}=Y_{v}^{r} Y_{\omega}^{i}-Y_{v}^{i} Y_{\omega}^{r}$. The angle $\alpha_{v, n b}$ is given by: $\alpha_{\mathrm{v}, \mathrm{nb}}=\operatorname{angle}\left(\mathrm{Y}_{\mathrm{nb}}\right)-\operatorname{angle}\left(\mathrm{Y}_{\mathrm{v}}\right)$. Therefore, the determinant depends on the derivatives $Y_{v}, Y_{\omega}$ of the individual oscillator and the parameter $Y_{n b}$ (parameter $Y_{12}$ of the $2 \times 2$ admittance matrix of the coupling network). By setting $\operatorname{det}[\mathrm{JC}]=0$, one obtains the following analytical condition for the D-type bifurcation locus in the plane $\phi_{1,2}, \phi_{2,3}$ :

$$
\begin{aligned}
& \sin \left(\phi_{2,3}-\phi_{1,2}\right) \sin \left(2 \alpha_{\mathrm{v}, \mathrm{nb}}\right)+ \\
& +\cos \left(\phi_{2,3}+\phi_{1,2}\right)\left(3-4 \sin ^{2} \alpha_{\mathrm{v}, \mathrm{nb}}\right)+ \\
& +\cos \left(\phi_{2,3}-\phi_{1,2}\right)\left(1+2 \cos ^{2} \alpha_{\mathrm{v}, \mathrm{nb}}\right)=0
\end{aligned}
$$

An identical result is obtained from the characteristic equation (12), taking into account that at a D-type bifurcation this equation must have a root of value zero, in addition to the one associated to the system autonomy (represented by the common factor $s$ ). Thus, the condition for D-type bifurcation is $\mathrm{p}_{1}=0$, which provides the same analytical expression (23). From inspection of (25), a fundamental result is derived: to the first-order approximation considered through the paper, the locus of D-type bifurcations only depends on the angle $\alpha_{\mathrm{v}, \mathrm{nb}}$. Therefore, once the angle $\alpha_{v}$ of the voltage derivative $Y_{v}$ is known, one can always synthesize the coupling network so as to obtain particular D-type bifurcation loci, which would be done in a straightforward manner. This property is believed to be highly relevant for the designer, since it enables a remarkable control on the global stability of the coupled system. It will be numerically validated in the next section, using both a numerical resolution of $\operatorname{det}[\mathrm{JC}]=0$ and a more involved extension of the nonlinear-simulation technique in [10]-[13].

Condition (25) can be particularized to the case of a constant phase shift progression $\phi_{1,2}=\phi_{2,3}=\phi$. In this case, the work [14] demonstrated that, under weak coupling conditions, the stable phase-shift range corresponds to $\left(-90^{\circ}, 90^{\circ}\right)$ when the condition $\cos \alpha_{\mathrm{v}, \mathrm{nb}}<0$ is fulfilled. This is true provided the system is not operating in the neighborhood of $\cos \alpha_{\mathrm{v}, \mathrm{nb}}=0$. If the opposite condition 
$\cos \alpha_{\mathrm{v}, \mathrm{nb}}>0$ is fulfilled, the stable phase-shift range is $\left(90^{\circ}\right.$, $270^{\circ}$ ), which, again, is true provided the system is not in the neighborhood of $\cos \alpha_{\mathrm{v}, \mathrm{nb}}=0$. As shown in [14], this stability inversion occurs continuously, but very rapidly as condition $\cos \alpha_{\mathrm{v}, \mathrm{nb}}=0$ is approached, such that the stable constant phase shift interval becomes zero at $\cos \alpha_{\mathrm{v}, \mathrm{nb}}=0$. The work [14] also demonstrated that optimum behavior of the coupled system with constant phase shift progression is obtained for $\sin \alpha_{\mathrm{v}, \mathrm{nb}}=0$, which leads to minimum variation of the oscillation frequency and the phase-noise spectral density with the constant inter-stage phase shift $\phi$. When particularizing (25) to the case $\phi=\phi_{1,2}=\phi_{2,3}$, one obtains the following condition:

$$
\cos (2 \phi)=\frac{2 \cos ^{2} \alpha_{\mathrm{v}, \mathrm{nb}}+1}{1-4 \cos ^{2} \alpha_{\mathrm{v}, \mathrm{nb}}}
$$

From inspection of (26), in the constant inter-stage phaseshift progression, D-type bifurcations can only occur for $\left|\cos \alpha_{\mathrm{v}, \mathrm{nb}}\right|=1$ or $\cos \alpha_{\mathrm{v}, \mathrm{nb}}=0$. In all the other cases, the term on the right hand side of (26) has magnitude bigger than one. The two possibilities correspond to two different situations. First one, $\left|\cos \alpha_{\mathrm{v}, \mathrm{nb}}\right|=1$, is in correspondence with the condition for optimum behavior: $\sin \alpha_{\mathrm{v}, \mathrm{nb}}=0$. In this situation, equation (26), satisfied at each of the two phase shift values $\phi=-90^{\circ}$ and $\phi=90^{\circ}$, provides the limits of the stable behavior interval. On the other hand, $\cos \alpha_{\mathrm{v}, \mathrm{nb}}=0$ is the limit condition for stability inversion [14].

To derive an analytical expression of the Hopf locus, one should apply the Fourier transform to system (8), which provides the following characteristic determinant:

$$
\operatorname{det}[\mathrm{j} \Omega[\mathrm{I}]-[\mathrm{M}]]=0
$$

where $\Omega$ is the perturbation frequency. After elimination of common factor $\mathrm{j} \Omega$, associated to the system autonomy, the analytical expansion of (26) leads to a cumbersome complex expression of fifth order in the frequency $\mathrm{j} \Omega$. From a careful inspection of this expression, it is gathered that, unlike the Dtype locus, the Hopf locus depends on $\left|\mathrm{Y}_{\mathrm{v}}\right|,\left|\mathrm{Y}_{\mathrm{nb}}\right|$ and the two angles $\alpha_{\mathrm{v}, \mathrm{nb}}$ and $\alpha_{\mathrm{v}, \omega}=\operatorname{angle}\left(\mathrm{Y}_{\omega}\right)-\operatorname{angle}\left(\mathrm{Y}_{\mathrm{v}}\right)$. As will be shown in the next section, the Hopf locus will not present a complex geometry, unless the system operates under small values of $\left|\cos \alpha_{v, n b}\right|$ and $\left|\sin \alpha_{v, \omega}\right|$. Note that a small negative value of $-\sin \left(\alpha_{\mathrm{v}, \omega}\right)$ implies a small stability margin of the individual oscillator in free-running conditions [12] , [27]). As expected, as $\sin \alpha_{\mathrm{v}, \omega}$ approaches zero, the plane $\phi_{1,2}, \phi_{2,3}$ becomes progressively void of stable points.

In systems exhibiting both Hopf and D-type bifurcations (like the coupled-oscillator system), there will be, in general, some particular points of the parameter space where two real eigenvalues cross through zero simultaneously. At these double-zero eigenvalue bifurcations [18], of co-dimension two, the Hopf locus and the D-type locus merge. In fact, the double zero eigenvalue can be interpreted as a degenerate Hopf bifurcation, where the frequency of the two critical poles $\pm \mathrm{j} \Omega$ becomes zero. Using the characteristic equation (12), these bifurcation points simultaneously fulfill (25) and the following condition:

$$
\mathrm{p}_{2}\left(\phi_{12}, \phi_{23}\right)=0
$$

The coefficient $\mathrm{p}_{2}$ has a very complex analytical expression. However, the distinct intersection points of (25) and (28) are mainly determined by the angle $\alpha_{\mathrm{v}, \mathrm{nb}}$, unless operating near the stability inversion (condition $\cos \alpha_{\mathrm{v}, \mathrm{nb}}=0$ ) where they be become very sensitive to other magnitudes, i.e., $\mathrm{Y}_{\mathrm{v}}, \mathrm{Y}_{\omega}$. This will be numerically confirmed in the practical example of Section IV.

\section{BIFURCATION ANALYSIS OF A COUPLED-OSCILLATOR SYSTEM AT $3.85 \mathrm{GHz}$}

The above formulation and bifurcation analysis has been applied to a practical system of $N=3$ oscillators operating at 3.85 GHz. The oscillators are designed using a field-effect transistor (NE3210S01) on a RO4003C substrate [Fig. 1(c)]. A varactor diode (SMV1232) is used as a tuning element for the control of the phase shift. A comparison between the simulated and experimental frequency versus tuning voltage curve of the individual oscillator (in free-running regime) is presented in Fig. 2. Although library models provided by the manufacturers have been used for all the lumped elements in simulation, some discrepancies between measurements and simulations have been found. A frequency offset of about 200 $\mathrm{MHz}$ is appreciable in Fig. 2 between the two curves. These discrepancies are attributed to the limited accuracy of the component models, tolerances of the etching process, and typical component dispersion in hybrid technology. The coupling networks are composed of a transmission line section of electrical lengths $\theta_{12}$ and $\theta_{23}$, with characteristic impedance $Z_{c}=100 \Omega$, bounded by series resistors of value $R_{c}=300 \Omega$ which corresponds to weak-coupling operation [14], [15]. Different values of $\theta_{12}$ and $\theta_{23}$ will be considered in this investigation.

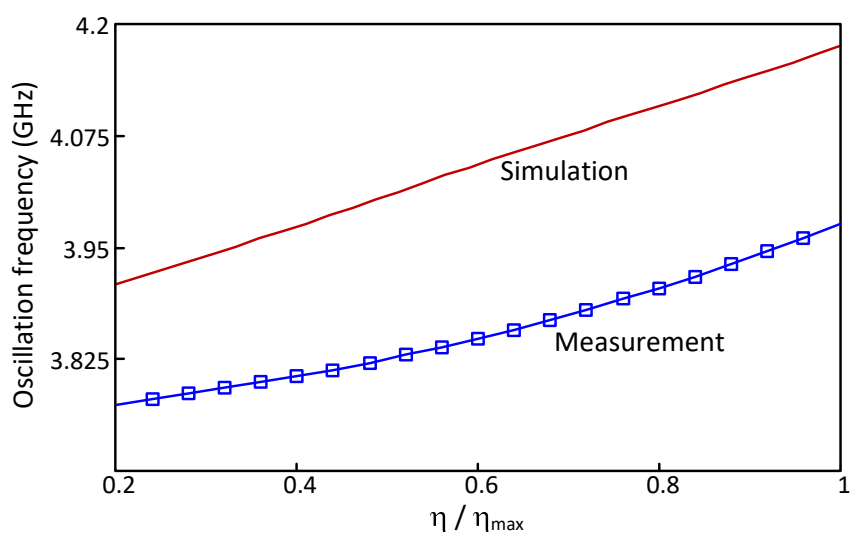


Fig. 2. Frequency versus normalized tuning voltage of the individual oscillator. Simulation is plotted in solid line and measurement results are plotted with squares.

Usually, the bifurcation loci of nonlinear circuits are traced in the plane defined by the system parameters, which in the case of the coupled system, correspond to the tuning voltages. However, this representation is ambiguous due to the coexistence, for the same parameter values, of solutions, with different phase values, as shown in [11]. Instead, a representation in the plane defined by the two phase-shift values $\phi_{1,2}=\phi_{2}-\phi_{1}$ and $\phi_{2,3}=\phi_{3}-\phi_{2}$ is carried out here. The bifurcation analysis considers all possible values of $\phi_{1,2}$ and $\phi_{2,3}$ and, of course, includes the particular case of a constant phase-shift distribution $\phi=\phi_{1,2}=\phi_{2,3}$ where the $\phi$ value is varied by detuning the outermost oscillators. In the plane defined by the two phase shifts $\phi_{1,2}, \phi_{2,3}$, the constant phase shift solution $\phi=\phi_{1,2}=\phi_{2,3}$ is defined by a straight line with unit slope.

In Sub-Section IV.A, the new analytical expression of the D-type locus will be validated through independent numerical resolutions of $\operatorname{det}[\mathrm{JC}]=0$ and through an extension to the case of a non-constant inter-stage phase shift of the already wellestablished numerical method in [10]-[13]. In the next SubSections IV.B, IV.C and IV.D, the system will be analyzed for three different electrical length values of the transmission line in the coupling network. The values chosen are $345^{\circ}, 363.5^{\circ}$ and $273.5^{\circ}$. These values have been selected taking into account the coupled-system performance when providing the solution with constant phase-shift progression [14]. First value $\left(\theta_{12}=\theta_{23}=345^{\circ}\right)$ is not optimum but satisfies the condition $\cos \alpha_{\mathrm{v}, \mathrm{nb}}<0$ for the stable constant phase-shift interval $\left(-90^{\circ}, 90^{\circ}\right)$. Second value $\left(\theta_{12}=\theta_{23}=363.5^{\circ}\right)$ is optimum for the stable interval $\left(-90^{\circ}, 90^{\circ}\right)$, as it fulfills $\sin \alpha_{\mathrm{v}, \mathrm{nb}}=0$ and $\cos \alpha_{\mathrm{v}, \mathrm{nb}}<0$ [14]. Third value $\theta_{12}=\theta_{23}=273.5^{\circ}$ ) satisfies the condition $\cos \alpha_{\mathrm{v}, \mathrm{nb}}>0$, which provides the stable constant phase-shift interval $\left(90^{\circ}, 270^{\circ}\right)$. The values $345^{\circ}$ and $273.5^{\circ}$ have been calculated after measuring the experimental oscillation frequency of the coupled system. For completeness, two additional electrical length values are considered in SubSections IV.E and IV.F, respectively corresponding to $\theta_{12}=$ $\theta_{23}=183.6^{\circ}$ and $\theta_{12}=\theta_{23}=100.1^{\circ}$. The first value $183.6^{\circ}$ fulfils $\sin \alpha_{\mathrm{v}, \mathrm{nb}}=0$ and $\cos \alpha_{\mathrm{v}, \mathrm{nb}}>0$, which gives rise to minimum oscillation frequency deviation through the stable constant inter-stage phase shift interval $90^{\circ}$ to $270^{\circ}$. The second value $100.1^{\circ}$ fulfills $\cos \alpha_{\mathrm{v}, \mathrm{nb}}=0$, corresponding to one of the two limit cases for stability inversion. Finally, in Sub-Section IV.G the case of two different electrical length values $\theta_{12} \neq \theta_{23}$ is considered.

\section{A. D-type loci for identical values of the angle $\alpha_{\mathrm{v}, \mathrm{nb}}$}

Initially, the validity of the analytical expression in (25) will be demonstrated through exhaustive comparison with numerical calculations of the D-type bifurcation loci using $\operatorname{det}[\mathrm{JC}]=0$ and an extension of the approach in [10]. Twenty different values of the angle $\alpha_{v}$ (angle of the oscillator derivative $Y_{v}$ ) have been considered, defined by $\alpha_{v}=\mathrm{m} 360^{\circ} / 20$, where the integer $\mathrm{m}$ goes from 0 to 19 . For each $\alpha_{\mathrm{v}}$, the electrical length $\theta_{12}=\theta_{23}=\theta$ of the transmission line in the coupling network is calculated, so as to have an identical $\alpha_{\mathrm{v}, \mathrm{nb}}$ value $\left(\alpha_{\mathrm{v}, \mathrm{nb}}=22.12^{\circ}\right)$ in all cases. This value agrees with the one corresponding to our actual oscillator derivative $Y_{v}$ and the electrical length $345^{\circ}$, considered in the next sub-section. As shown in Fig. 3(a), all the D-type bifurcation loci obtained through numerical resolution of $\operatorname{det}[\mathrm{JC}]=0$ are overlapped (20 curves) and exactly agree with the locus provided by the analytical expression in (25). The results obtained through the extension of numerical method in [10]-[13] (20 curves) have also been superimposed and show an excellent agreement. Note that in the case of non-constant inter-stage phase shifts, convergence problems are often encountered with that numerical formulation, especially for $\alpha_{\mathrm{v}, \mathrm{nb}}$ values near the stability inversion. The results in Fig. 3(a) demonstrate the correctness of the analytical expression (25).

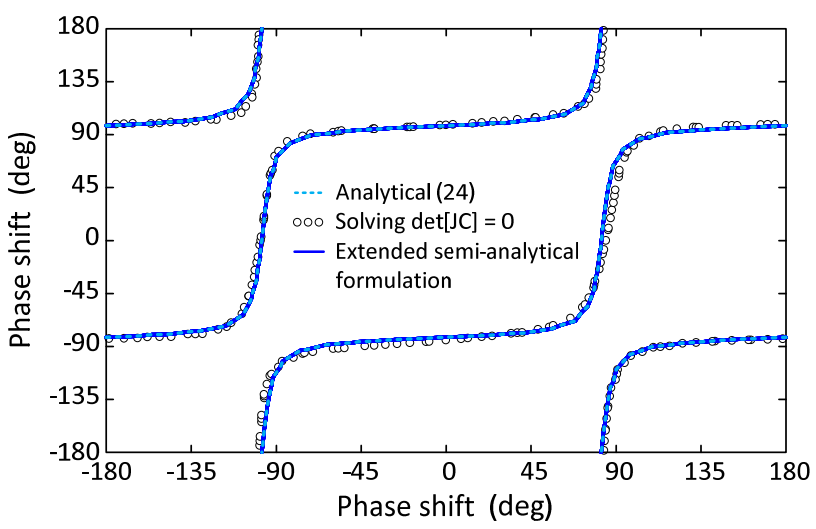

(a)

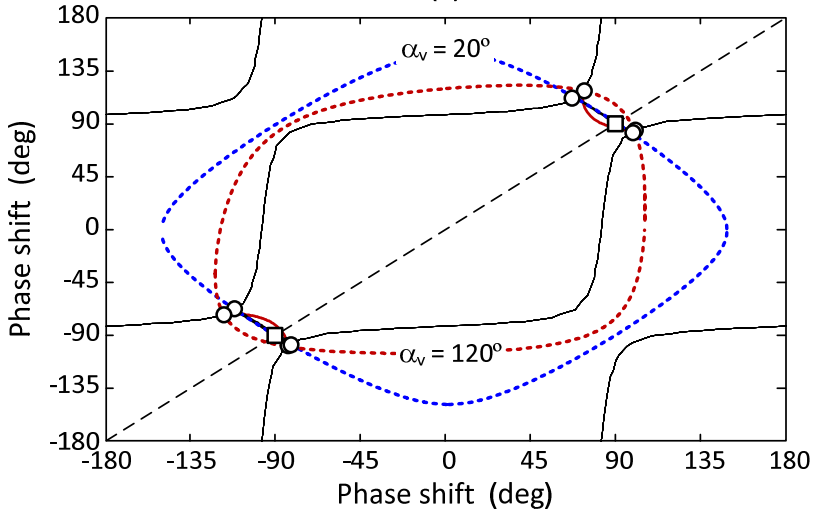

(b)

Fig. 3 (a) Ensemble of D-type bifurcation loci obtained for twenty different values of electrical length and angle $\alpha_{\mathrm{v}}$, but maintaining a constant value of the angle $\alpha_{\mathrm{v}, \mathrm{nb}}=22.12^{\circ}$. Superimposed are the results of the new analytical expression (25), the numerical resolution of $\operatorname{det}[\mathrm{JC}]=0$ and an extension of the simulation technique in [10]. (b) D-type bifurcation locus obtained from (25) (solid line) and locus of points fulfilling condition (28) (dashed lines), for $\alpha_{v}=20^{\circ}$ and 
$\alpha_{v}=120^{\circ}$. The two Hopf bifurcation points of the constant phaseshift solutions are plotted with squares.

Note that the stable regions are also determined by the Hopf bifurcation locus, which has not been considered yet. However, some points of this locus are readily available if we take into account the following. Unless the system operates near the stability inversions, and under weak coupling conditions, the edges of the stable interval of the constant phase shift progression will be given by $-90^{\circ}$ and $90^{\circ}$, which (except for $\sin \alpha_{\mathrm{v}, \mathrm{nb}}=0$ ) should correspond to Hopf bifurcations. On the other hand, the double-zero eigenvalue bifurcations provide the points where the Hopf locus and the D-type locus merge. They are obtained from the intersections of the D-type locus with the locus of $\phi_{1,2}, \phi_{2,3}$ values fulfilling (28).

The D-type locus given by (25) and the locus composed by the points fulfilling (28) have been traced in Fig. 3(b) for two different values of $\alpha_{\mathrm{v}}\left(20^{\circ}\right.$ and $\left.120^{\circ}\right)$, calculating in each case the electrical length $\theta_{12}=\theta_{23}=\theta$ so as to have the same value of the angle difference $\alpha_{\mathrm{v}, \mathrm{nb}}=22.12^{\circ}$, considered in the previous analysis. The double-zero eigenvalue bifurcations, obtained from the intersections of (25) and (28), are marked with circles. As can be seen in Fig. 3(b), the intersection points (double-zero eigenvalue bifurcations) for the two $\alpha_{\mathrm{v}}$ values $\left(20^{\circ}\right.$ and $\left.120^{\circ}\right)$ are very close. The Hopf bifurcations corresponding to the solution with constant inter-stage phase shift are marked with squares in the same figure.

\section{B. Electrical length $\theta_{12}=\theta_{23}=345^{\circ}$}

Initially, the electrical length $\theta_{12}=\theta_{23}=345^{\circ}$ has been considered, obtaining the loci of Fig. 3. For better illustration of the patterns of the Hopf and D-type bifurcation loci, these loci have been represented in the interval $-360^{\circ}$ to $360^{\circ}$ in Fig. $4(\mathrm{a})$. The set of phase points $\phi_{1,2}, \phi_{2,3}$ at which one real eigenvalue crosses the imaginary axis through zero (zeroeigenvalue or D-type locus) is traced with solid line. The set of phase points at which a pair of complex-conjugate eigenvalues crosses the imaginary axis (Hopf-bifurcation locus) is traced with dashed line. The two loci delimit sections of the phase plane with different stability properties. By performing complementary local stability analyses in the various sections, using the formulation (8), it has been determined that the shaded region (Region A in Fig. 3) is stable, with all the eigenvalues on the left-hand side of the complex plane. This stable region is bounded by the zeroeigenvalue locus and the Hopf locus.

As shown in Fig. 4(a), the Hopf locus is composed of two curves: Hopf-1 and Hopf-2. In similar manner, the D-type locus is composed by two curves Zero-1 and Zero-2, each containing two sections (Zero-1a, Zero-1b) and (Zero-2a, Zero-2b). The curve Hopf-1 arises at one end from the merging of Zero-1a and Zero-1b and at the other end from the merging of Zero-2a and Zero-2b. The same is valid for the curve Hopf-2. At each merging point, the system has two eigenvalues at zero. As already stated, the point with double zero eigenvalue is a co-dimension two bifurcation. From the merging point and along the Hopf-bifurcation locus, the imaginary part $\Omega$ of the two eigenvalues $(\sigma \pm \mathrm{j} \Omega$, where $\sigma=0)$ grows from zero. Between the curves Zero-1 and Zero-2 [Region B in Fig. 4(a)], the system contains one real eigenvalue on the right hand side of the complex plane. Therefore, it is unstable. Between the sections Hopf-1, Hopf2, Zero- $1 \mathrm{~b}$ and Zero-2b corresponding to Region $\mathrm{C}$ in Fig. 4(a), the system has two unstable eigenvalues, either real or complex-conjugate. In summary, there are three zones in the diagram: A, B and C. Region A is stable, Region B corresponds to solutions with one unstable eigenvalue and Region C corresponds to solutions with two unstable eigenvalues.

Fig. 4(b) shows the representation of the loci limited to the interval $-180^{\circ}$ to $180^{\circ}$ in the two phase-shift variables $\left(\phi_{1,2}, \phi_{2,3}\right)$. Note that in agreement with the fulfillment of $\cos \alpha_{\mathrm{v}, \mathrm{nb}}<0$, the stable range of the constant phase-shift solution path $\phi=\phi_{1,2}=\phi_{2,3}$ [straight line of slope 1 in Fig. $4(\mathrm{~b})$ ] corresponds to $\left(-90^{\circ}, 90^{\circ}\right)$. The stable region is not totally symmetrical about the constant phase shift solution, exhibiting a larger stable zone above this line. As shown in the next sub-section, symmetry about $\phi=\phi_{1,2}=\phi_{2,3}$ requires a specific (optimum) design of the coupling network.

In order to validate the analysis results, phase measurements of the oscillator array have been performed. A four-channel digital oscilloscope (Infiniium DSO90804A) with a bandwidth of $8 \mathrm{GHz}$ and a sample rate of $40 \mathrm{GSa} / \mathrm{s}$ has been used for this purpose. The three outputs of the oscillator array are routed through coaxial cables to three channels of the oscilloscope. A phase calibration is previously carried out to account for any delay difference between the three measurement channels (including the external cabling). Simultaneous FFT is applied to the three channels to get the phase-shifts $\phi_{1,2}$ and $\phi_{2,3}$ between the fundamental components of the three oscillators. Then, the experimental loci are obtained delimiting the border between synchronization and desynchronization as the tuning parameters (varactor bias voltages) are varied. Measurement results for the case $\theta_{12}=\theta_{23}=345^{\circ}$ are superimposed in Fig. 4(b). The measurement uncertainty associated to the oscillator phase-shift measurements has been estimated to be about $\pm 5^{\circ}$, after compensation of the delay differences among channels. Considering this measurement uncertainty, plus the finite accuracy of the electrical models and the technological dispersion typical in hybrid prototypes, there is a reasonable agreement between the experimental results and the bifurcation loci predicted with the new method in Fig. 4(b).

Besides the use of the digital oscilloscope, one of the oscillator outputs is connected to a spectrum analyzer in the measurement setup. This enables the distinction between $\mathrm{D}-$ type [squares in Fig. 4(b)] and Hopf [crosses in Fig. 4(b)] bifurcations. The two different types are distinguished from the qualitative differences in the measured spectrum of the quasi-periodic solution (resulting from the bifurcation) obtained immediately after this bifurcation. At a D-type bifurcation, the quasi-periodic solution at the two fundamental frequencies $\omega$ and $\Omega$, originates from zero value of the difference frequency $\Delta \omega=|\omega-\Omega|$. This is in agreement with 
the fact that a real eigenvalue passes through zero at this $\mathrm{D}$ type bifurcation point [12]. Therefore, immediately after the bifurcation, a dense spectrum is observed, such as the one in Fig. 5(a) corresponding to the bifurcation point labeled $P_{D}$ in Fig. 4(b). On the other hand, at a Hopf bifurcation the quasiperiodic solution at $\omega$ and $\Omega$ is generated from frequency difference $\Delta \omega \neq 0$ and zero amplitude at the incommensurable frequency $\Omega$ [12]. An example, corresponding to the bifurcation point labeled $\mathrm{P}_{\mathrm{H}}$ in Fig. 4(b), is shown in Fig. 5(b). Most of the measured points correspond to D-type bifurcation [Fig. 4(b)], with the Hopf-bifurcation points closing the two sections of the D-type bifurcation locus at the two codimension two points.

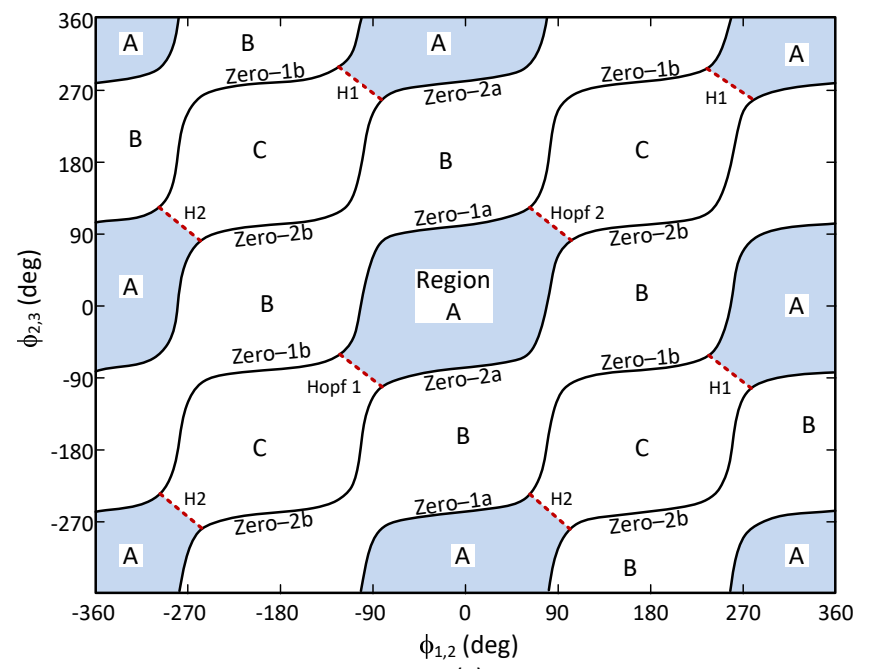

(a)

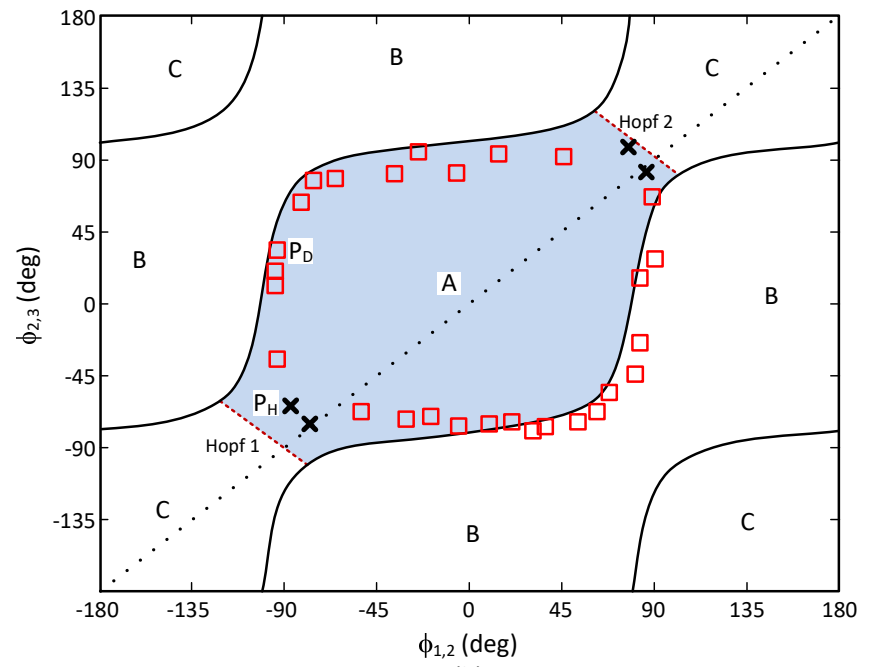

(b)

Fig. 4. Bifurcation loci for coupling-line electrical length $\theta=345^{\circ}$. The shaded region is stable. $\mathrm{D}$-type locus is traced with solid line. Hopf locus is traced with dashed line. (a) Bifurcation loci traced in the interval $-360^{\circ}$ to $360^{\circ}$, for better illustration of the patterns. (b) Same bifurcation loci represented in the interval $-180^{\circ}$ to $180^{\circ}$. Superimposed are the measured D-type (squares) and Hopf (crosses) bifurcation loci. The constant-phase shift solutions correspond to a straight line of unit slope, represented with dotted line.

The above result has been validated through bifurcation analysis versus a single analysis parameter, corresponding to the tuning voltage $\Delta \eta_{2}$. For illustration, the pair of tuning voltages $\Delta \eta_{1 \mathrm{o}}=-0.216 \mathrm{~V}, \Delta \eta_{2 \mathrm{o}}=0.340 \mathrm{~V}$ that provides the constant phase shift $\phi_{1,2}=\phi_{2,3}=90^{\circ}$ has been initially selected. Then, the tuning voltage $\Delta \eta_{1}$ is kept fixed at $\Delta \eta_{1}=\Delta \eta_{10}=-0.216 \mathrm{~V}$. Next, the frequency deviation for all the solutions of system (1) is represented versus $\Delta \eta_{2}$. This provides the closed multi-valued curve of Fig. 6(a). The two phase shift values $\phi_{1,2}, \phi_{2,3}$ vary along this closed curve and the equality $\phi_{1,2}=\phi_{2,3}$ is fulfilled only at the two points marked with large circles. Point $\Delta \eta_{10}=-0.216 \mathrm{~V}, \Delta \eta_{20}=0.340 \mathrm{~V}$ corresponds to the constant phase-shift solution $\phi_{1,2}=\phi_{2,3}=90^{\circ}$. Point $\Delta \eta_{10}=-0.216 \mathrm{~V}$, $\Delta \eta_{20}=0.065 \mathrm{~V}$ corresponds to a second constant phase-shift solution $\phi_{1,2}=\phi_{2,3}=149.6^{\circ}$. Four turning points labeled $T_{1}$ to $\mathrm{T}_{4}$ are shown in the curve of Fig. 6(a).

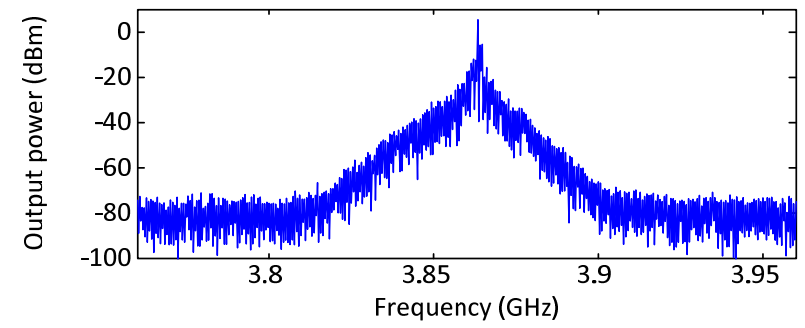

(a)

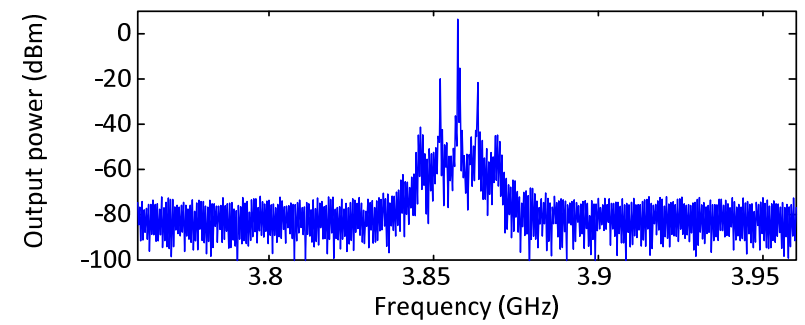

(b)

Fig. 5. Distinction between D-type and Hopf bifurcations with spectra measured immediately after the bifurcation. (a) D-type bifurcation. Quasi-periodic solution originates from zero value of the difference frequency $\Delta \omega=\left|\omega_{0}-\Omega\right|$. (b) Hopf bifurcation. Quasiperiodic solution originates at $\Delta \omega \neq 0$ from zero amplitude of the autonomous components.

Next, the real parts of the six eigenvalues of the matrix [M] in (8) have been traced versus $\Delta \eta_{2}$ at constant $\Delta \eta_{1}=\Delta \eta_{10}=-0.216 \mathrm{~V}$, with the results presented in Fig. 6(b). For better clarity, only the three dominant eigenvalues are shown. One of these eigenvalues is $\lambda_{1}=0$, associated to the solution autonomy. Section $\mathrm{S}_{1}-\mathrm{S}_{2}$ [in Fig. 6(b)] has two complex-conjugate eigenvalues $\lambda_{2}, \lambda_{3}=\lambda_{2}^{*}$. At each of the two points $\mathrm{S}_{1}$ and $\mathrm{S}_{2}$ the pair of complex-conjugate eigenvalues splits into two real eigenvalues $\lambda_{2}, \lambda_{3} \in \mathrm{R}$. At each of the four turning points $T_{1}$ to $T_{4}$ in Fig. 6 (b), a real eigenvalue passes through the imaginary axis. Section $\mathrm{H}-\mathrm{S}_{1}$ is stable with a pair of complex-conjugate eigenvalues on the left-hand side of the complex plane (LHP) [Fig. 6(b)]. Next 
the evolution of the two eigenvalues $\lambda_{2}, \lambda_{3}$ when varying $\Delta \eta_{2}$ in the sense of the arrows [indicated in Fig. 6(a) and Fig. 6(b)] will be described in detail. At $\mathrm{S}_{1}$, the pair of complexconjugate eigenvalues splits into two real eigenvalues $\lambda_{2}, \lambda_{3} \in \mathrm{R}$. At $\mathrm{T}_{1}, \lambda_{2}$ passes through zero, so the section $\mathrm{T}_{1-}$ $T_{2}$ is unstable. At $T_{2}$, the same eigenvalue crosses again to the LHP, so the section $T_{2}-T_{3}$ is stable. At $T_{3}$, the eigenvalue $\lambda_{2}$ passes through zero again, so the section $T_{3}-T_{4}$ is unstable with one real eigenvalue on the right-hand side of the complex plane (RHP). At $\mathrm{T}_{4}$, the real eigenvalue $\lambda_{3}$ also crosses to the RHP. Between $T_{4}$ and $S_{2}$, the solution curve has two real eigenvalues on the RHP. At $\mathrm{S}_{2}$, they merge into two complexconjugate eigenvalues $\lambda_{2}, \lambda_{3}=\lambda_{2}^{*}$ on the RHP. At the Hopf bifurcation $\mathrm{H}$, these two complex-conjugate eigenvalues cross the imaginary axis to the LHP, so the section $\mathrm{H}-\mathrm{T}_{1}$ is stable.

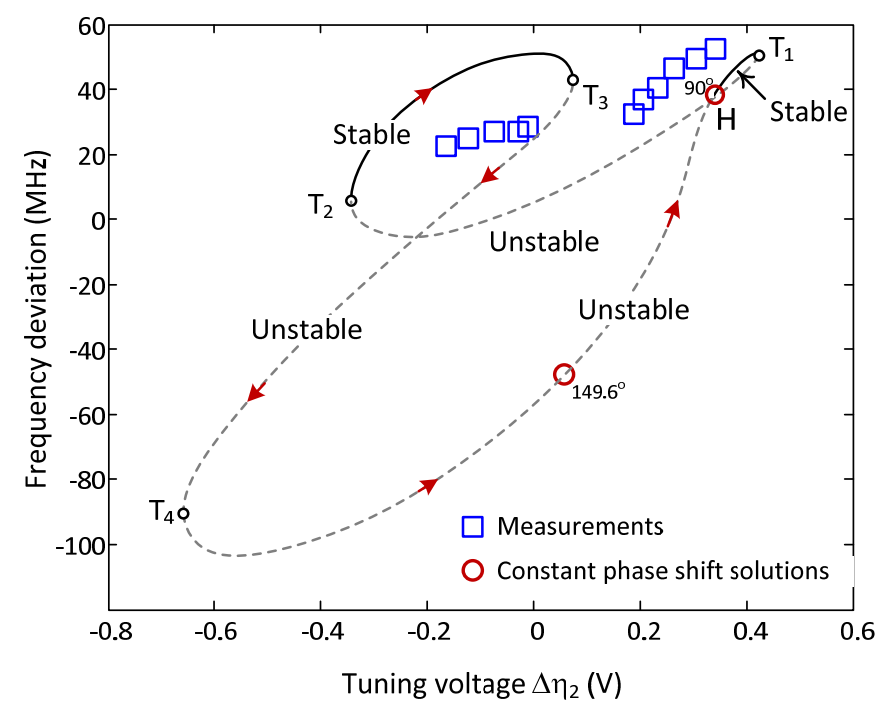

(a)

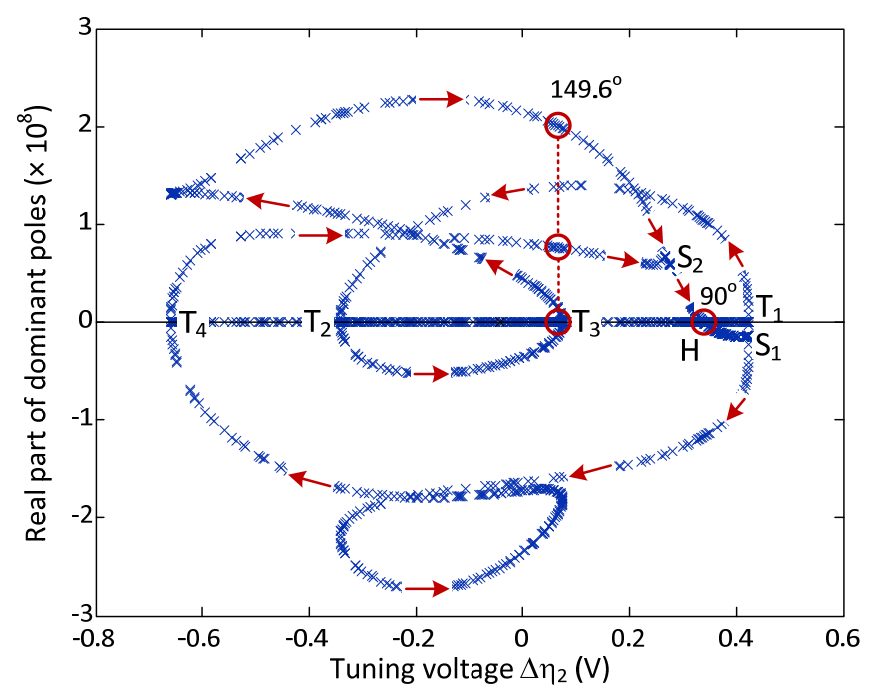

(b)

Fig. 6. Bifurcation analysis versus a single analysis parameter: the tuning voltage $\Delta \eta_{2}$, at constant $\Delta \eta_{1}=\Delta \eta_{10}=-0.216 \mathrm{~V}$. (a) Frequency deviation traced versus $\Delta \eta_{2}$. Measurements are superimposed with squares. (b) Variation of the real parts of the three dominant eigenvalues of the matrix [M] in (8) versus $\Delta \eta_{2}$. The points marked with circles correspond to the only two solutions with constant phase shift: $\phi_{1,2}=\phi_{2,3}=90^{\circ}$ and $\phi_{1,2}=\phi_{2,3}=149.6^{\circ}$.

In total agreement with the results in Fig. 4, the Hopf bifurcation $\mathrm{H}$ takes place at $\Delta \eta_{10}=-0.216 \mathrm{~V}$, $\Delta \eta_{20}=0.340 \mathrm{~V}$, corresponding to the constant phase-shift value $\phi_{1,2}=\phi_{2,3}=90^{\circ}$. Also in agreement with Fig. 4 , the solution with constant phase shift value $\phi_{1,2}=\phi_{2,3}=149.6^{\circ}$ is unstable with two real eigenvalues on the RHP [Region C of Fig. 4(b)]. This stability analysis versus the single parameter $\Delta \eta_{2}$ constitutes an independent validation of the new method.

The stable sections of the solution curve have been traced in solid line in Fig. 6(a). Measurements of the frequency deviation in the coupled-oscillator system are superimposed with squares. A reasonable agreement is found, given the original discrepancies in the individual (free-running) oscillator.

\section{Electrical length $\theta_{12}=\theta_{23}=363.5^{\circ}$}

Now the analysis is performed for the electrical length $\theta_{12}=\theta_{23}=\theta=363.5^{\circ}$. For this electrical length, the two conditions $\sin \alpha_{\mathrm{v}, \mathrm{nb}}=0$ and $\cos \alpha_{\mathrm{v}, \mathrm{nb}}<0$ are approximately fulfilled. As shown in [14], these two conditions enable an optimum system design for constant phase-shift progression $\phi=\phi_{1,2}=\phi_{2,3}$, with stable phase shift range $\left(-90^{\circ}, 90^{\circ}\right)$. Indeed, fulfillment of $\sin \alpha_{\mathrm{v}, \mathrm{nb}}=0$ implies minimum oscillation frequency deviation, maximum tuning range and minimum variation of the phase noise through this stable constant phase-shift interval [14]. In Fig. 7(a), the corresponding bifurcation loci are represented in the plane defined by $\phi_{1,2}, \phi_{2,3}$, considering the phase interval $-360^{\circ}$ to $360^{\circ}$, for better illustration of their patterns. In Fig. 7(b), the representation is limited to the interval $-180^{\circ}$ to $180^{\circ}$. The stable region $\mathrm{A}$, which has evolved into an approximate square, is bounded by the zero-eigenvalue loci Zero-1a and Zero-2a. Note that the vertical sections of these two loci are very sensitive to changes in the phase $\phi_{2,3}$, with very quick evolution under changes in this phase value. For the electrical length which exactly fulfils $\sin \alpha_{\mathrm{v}, \mathrm{nb}}=0$ and $\cos \alpha_{\mathrm{v}, \mathrm{nb}}<0$, the two sections of the Hopf locus would give rise to two codimension two bifurcations, located at the edges of the stable constant phase shift interval. No measurements are presented for this particular case since it is not possible, in practice, to achieve the precise electrical-length value fulfilling $\sin \alpha_{\mathrm{v}, \mathrm{nb}}=0$ and $\cos \alpha_{\mathrm{v}, \mathrm{nb}}<0$. 


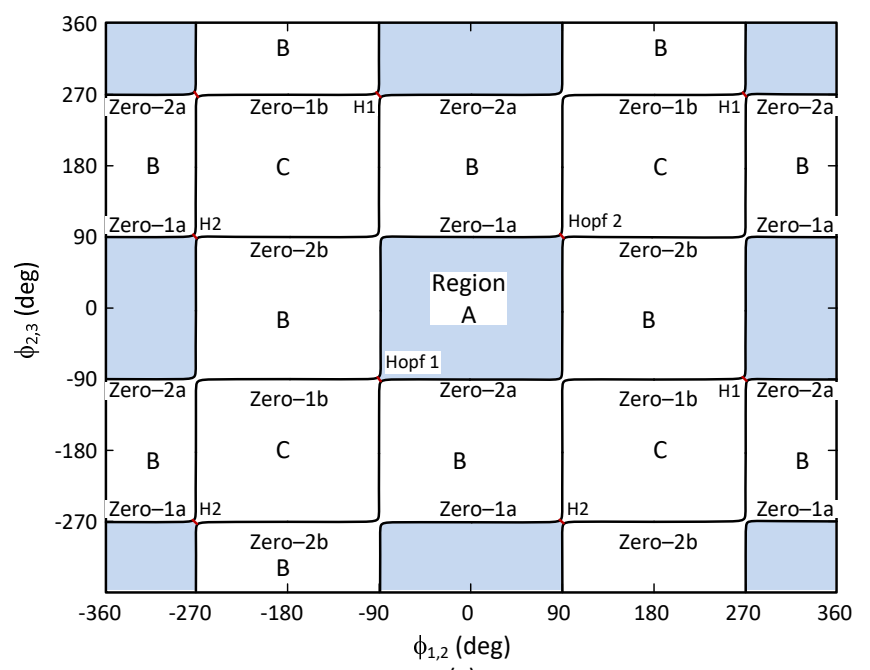

(a)

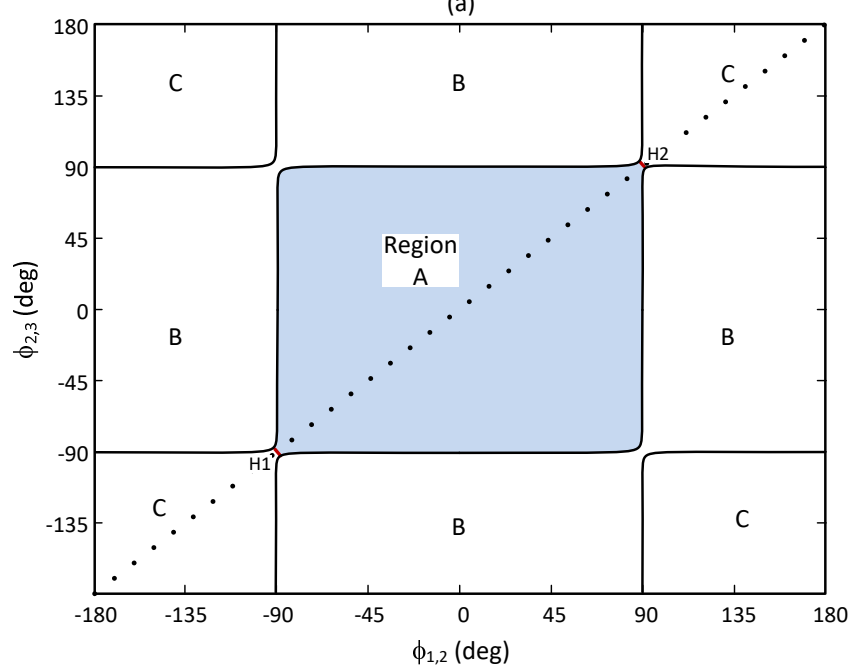

(b)

Fig. 7. Bifurcation loci for the optimal coupling-line electrical length $\theta=363.5^{\circ}$. The shaded region is stable. D-type locus is traced with solid line. The extremely small Hopf bifurcation locus is traced with dashed line. (a) Bifurcation loci traced in the interval $-360^{\circ}$ to $360^{\circ}$, for better illustration of the patterns. (b) Same bifurcation loci represented in the interval $-180^{\circ}$ to $180^{\circ}$. The constant-phase shift solutions correspond to a straight line of unit slope, represented with dotted line.

\section{Electrical length $\theta_{12}=\theta_{23}=273.5^{\circ}$}

Analysis results for $\theta_{12}=\theta_{23}=\theta=273.5^{\circ}$ are plotted in Fig. 8. The stable zone (region $\mathrm{A}$ ) and unstable zones with two unstable eigenvalues (region C) are inverted [Fig. 8(a)]. In fact, the stable region is located between the sections Hopf-1, Hopf-2, Zero- $1 \mathrm{~b}$ and Zero-2b. The region B, with one unstable real eigenvalue, preserves the original location, between the loci Zero-1 (Zero-1a plus Zero-1b) and Zero-2 (Zero-1a plus Zero-1b), which constitutes the two sections of the Dtype locus. Comparison between analysis and measurements is presented in Fig. 8(b), where the representation is restricted to the interval $-180^{\circ}$ to $180^{\circ}$. The constant phase shift solution [straight line of slope 1 in Fig. 8(b)] is stable between $90^{\circ}$ and $270^{\circ}$, in agreement with the fact that $\cos \alpha_{\mathrm{v}, \mathrm{nb}}>0$ for the electrical length $\theta=273.5^{\circ}$.

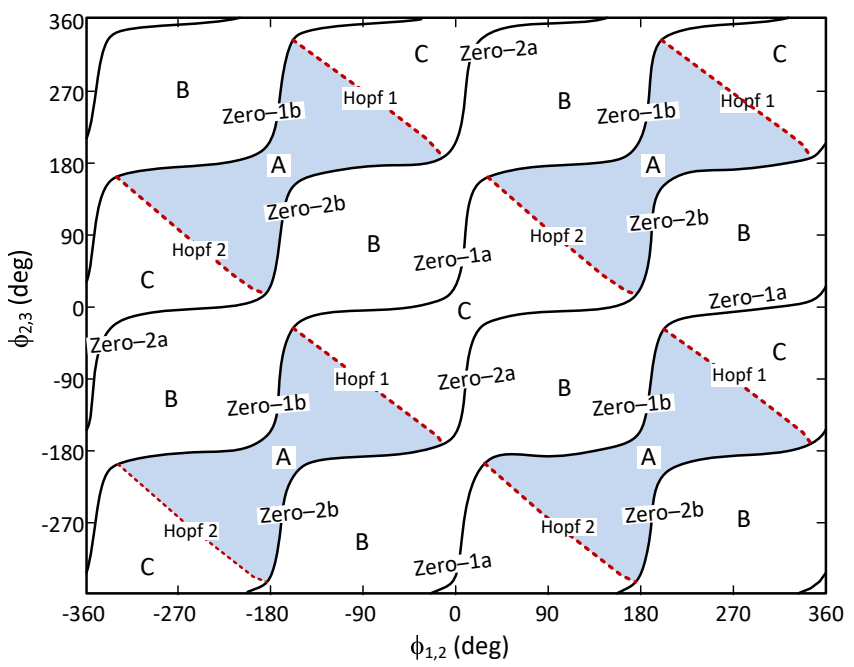

(a)

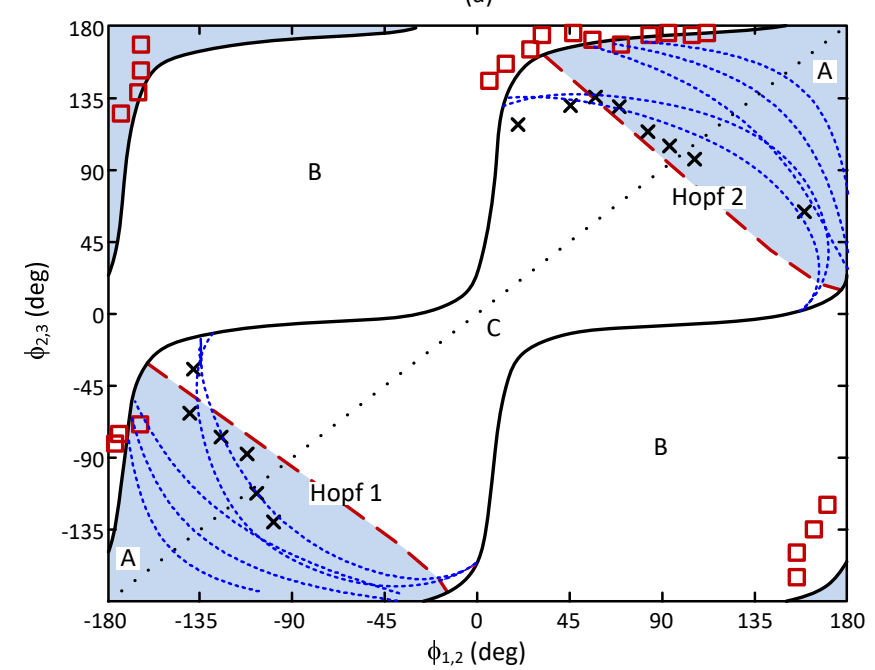

(b)

Fig. 8. Bifurcation loci for coupling-line electrical length $\theta=273.5^{\circ}$. The shaded region is stable. $\mathrm{D}$-type locus is traced with solid line. Hopf loci are traced with dashed line. (a) Bifurcation loci traced in the interval $-360^{\circ}$ to $360^{\circ}$, for better illustration of the patterns. (b) Same bifurcation loci represented in the interval $-180^{\circ}$ to $180^{\circ}$. Superimposed are the measured D-type (squares) and Hopf (crosses) bifurcation loci. The constant-phase shift solutions correspond to a straight line of unit slope, represented with dotted line. A set of Hopf loci obtained for arbitrary for values of $\alpha_{v, \omega}$ has also been traced with short dashed lines.

The analysis method is able to predict the stability inversion and the simulated bifurcation loci approximately delimit the stable region. However, agreement between simulation and measurements is worse than in the previous of case. This is attributed to the particular electrical length value, $\theta=273.5^{\circ}$, very close to the value fulfilling $\cos \alpha_{\mathrm{v}, \mathrm{nb}}=0$, given by $\theta=279.5^{\circ}$, at which a stability inversion takes place. As already discussed in Section III, near these stability inversions (occurring for $\alpha_{\mathrm{v}, \mathrm{nb}}=90^{\circ}$ and $\alpha_{\mathrm{v}, \mathrm{nb}}=270^{\circ}$ ) the Hopf locus becomes highly dependent on the angle $\alpha_{\mathrm{v}, \omega}$. This is illustrated in Fig. 8(b), where a set of Hopf loci obtained for arbitrary values of $\alpha_{v, \omega}$ is traced with short dashed lines. As can be observed, there is a significant variation of the pattern 
of the Hopf loci versus $\alpha_{\mathrm{v}, \omega}$. This high sensitivity to the angle $\alpha_{v, \omega}$ is only observed for $\alpha_{v, n b}$ values near the stability inversion. Note that the D-type locus does not change with $\alpha_{\mathrm{v}, \omega}$, as deduced from (25).

\section{E. Electrical length $\theta_{12}=\theta_{23}=183.6^{\circ}$}

For the electrical length $\theta_{12}=\theta_{23}=\theta=183.6^{\circ}$, the two conditions $\sin \alpha_{\mathrm{v}, \mathrm{nb}}=0$ and $\cos \alpha_{\mathrm{v}, \mathrm{nb}}>0$ are approximately fulfilled. As shown in [14], these two conditions enable an optimum system design for the constant phase-shift progression $\phi_{1,2}=\phi_{2,3}=\phi$, with the stable phase shift range $\left(90^{\circ}, 270^{\circ}\right)$. The corresponding bifurcation loci have been traced in Fig. 9(a). The global stability properties obtained when the above two conditions are fulfilled are reciprocal to the ones resulting from $\sin \alpha_{\mathrm{v}, \mathrm{nb}}=0$ and $\cos \alpha_{\mathrm{v}, \mathrm{nb}}<0$. The stable and unstable regions are inverted and the edges of the stable constant phase shift interval are delimited by doublezero eigenvalue bifurcations, in a manner analogous to the case of $\theta_{12}=\theta_{23}=\theta=363.5^{\circ}$.

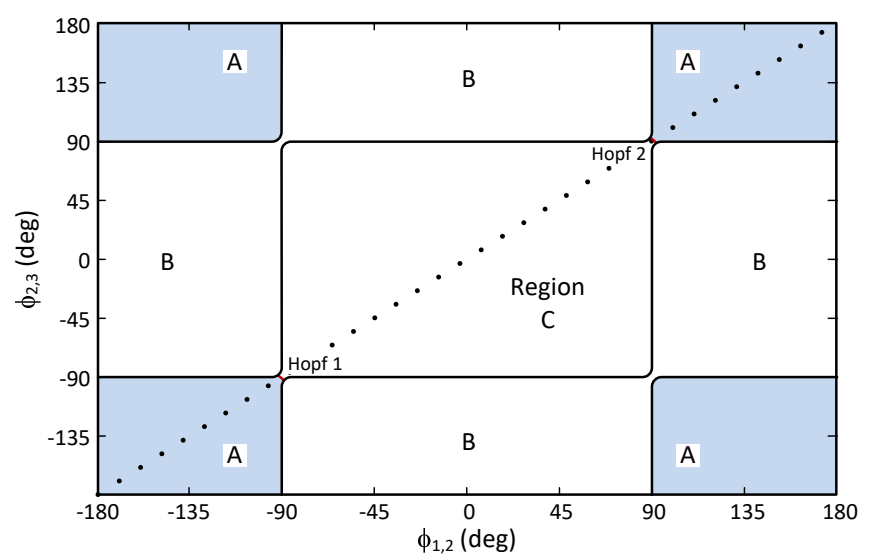

(a)

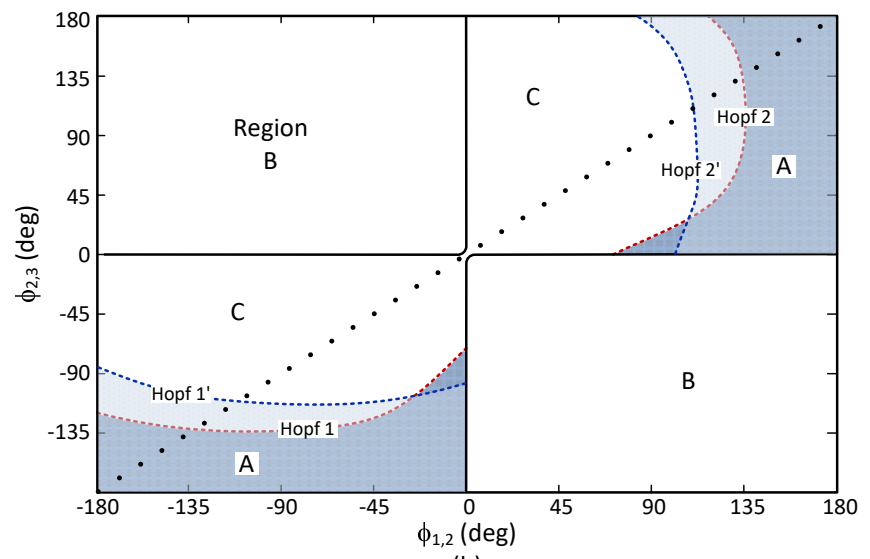

(b)

Fig. 9 Other electrical length values of theoretical interest. D-type loci are traced with solid line. Hopf loci are traced with dashed line. The shaded region is stable. The constant-phase shift solutions correspond to a straight line of unit slope, represented with dotted line. (a) $\theta_{12}=\theta_{23}=\theta=183.6^{\circ}$. (b) $\theta_{12}=\theta_{23}=\theta=100.1^{\circ}$. Neighborhood of the stability inversion. Superimposed a second Hopf locus, marked as Hopf', calculated after modifying $10^{\circ}$ the original value of $\alpha_{\mathrm{v}, \omega}$.

\section{F. Electrical length $\theta_{12}=\theta_{23}=100.1^{\circ}$}

For the electrical length $\theta_{12}=\theta_{23}=\theta=100.1^{\circ}$, the condition $\cos \alpha_{\mathrm{v}, \mathrm{nb}}=0$ is approximately fulfilled, which, as shown in [14], should give rise to a stability inversion of the solution with constant phase shift progression, changing from $\left(-90^{\circ}, 90^{\circ}\right)$ to $\left(90^{\circ}, 270^{\circ}\right)$ or vice versa, depending on the sense of variation of the parameters. As shown in [14], the inversion occurs continuously but very rapidly, and the stable phase interval (in the constant phase-shift progression) is zero for $\cos \alpha_{\mathrm{v}, \mathrm{nb}}=0$. It is so rapid that, although for $\theta=100.1^{\circ}$ we approach this condition to the first decimal figure, stable sections are observable in the constant phase shift straight line of Fig. 9(b). As already shown in Section IV.D, near the stability inversion the Hopf locus exhibits high sensitivity to the angle $\alpha_{\mathrm{v}, \omega}$. This is illustrated again in Fig. 9(b), where a second Hopf locus, marked as Hopf', has been calculated and superimposed after modifying $10^{\circ}$ the original value of $\alpha_{\mathrm{v}, \omega}$. We can observe how rather small changes in $\alpha_{\mathrm{v}, \omega}$ have a strong effect on the Hopf locus.

\section{G. Different electrical lengths}

The results in Fig. 4 to Fig. 9 have been obtained for the case of identical oscillators and coupling networks, with the same electrical lengths $\theta_{12}=\theta_{23}=\theta$. Here the impact of differences in the electrical length values will be investigated. In the system of $N=3$ oscillator elements, this will give rise to two different values of the angle $\alpha_{\mathrm{v}, \mathrm{nb}}$, so we can expect a big impact on the bifurcation behavior. Fig. 10 shows the result for two different values of electrical length: $\theta_{12}=345^{\circ}$ and $\theta_{23}=273^{\circ}$. For these two values, an inversion of the two Hopflocus sections is obtained. There is also a significant reduction of the stable range of the constant phase-shift solution, with two stable intervals, corresponding to $\left(-90^{\circ},-66^{\circ}\right)$ and $\left(20^{\circ}, 90^{\circ}\right)$, approximately (Fig. 10). Measurement results are superimposed in Fig. 10 showing reasonable agreement.

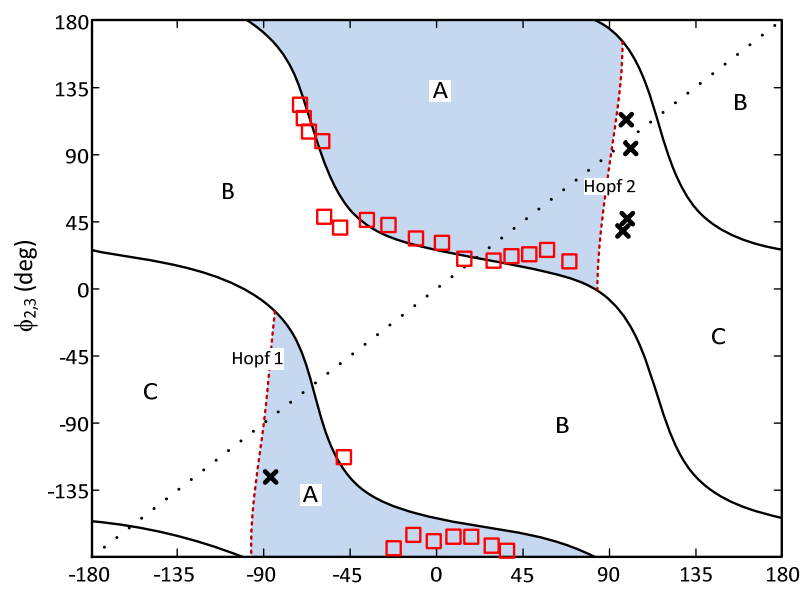

Fig. 10. Bifurcation loci for a coupled system with electrical length $\theta_{12}=345^{\circ}$ of the coupling line between the first and second oscillator and $\theta_{23}=273^{\circ}$ between the second and third oscillator elements. The shaded region is stable. $\mathrm{D}$-type locus is traced with solid line. Hopf locus is traced with dashed line. Superimposed are the measured Dtype (squares) and Hopf (crosses) bifurcation loci. The constant phase-shift solutions correspond to a straight line of unit slope, represented with dotted line. 


\section{Stability of Coexisting Steady-State Solutions}

As discussed in [3], in a coupled system with $\mathrm{N}$ oscillator elements operating in synchronized regime one can expect up to $2^{\mathrm{N}-1}$ solutions coexisting for each pair of tuning-parameter values. This can be understood as an extension of the two possible phase-shift values in an injection-locked oscillator (with an independent sinusoidal source), as shown in Adler's theory [28]. In the following, a method will be presented to determine the stability properties of all the steady-state solutions coexisting for a same pair of tuning element values $\Delta \eta_{1}, \Delta \eta_{2}$. In a manner similar to the methodology for the calculation of the bifurcation loci, the procedure will be based on the use of the inter-stage phase shifts as analysis parameters, instead of the actual system parameters $\Delta \eta_{1}, \Delta \eta_{2}$.

Due to the practical interest of the constant phase-shift distribution, the tuning-parameter values $\Delta \eta_{1}, \Delta \eta_{2}$ providing each constant phase-shift solution $\phi=\phi_{1,2}=\phi_{2,3}$ will be considered, obtaining exhaustively all the steady-state solutions that coexist with this solution. Note that for each $\phi$ value, a particular pair of tuning element values $\Delta \eta_{1}, \Delta \eta_{2}$ is necessary. The method has two stages. In a first stage, all the solutions curves $\phi_{1,2}, \phi_{2,3}$ coexisting with the one corresponding to the constant phase-shift progression $\phi=\phi_{1,2}=\phi_{2,3}$ will be obtained. In a second stage, their stability will be graphically determined by simply plotting the solution curves $\phi_{1,2}, \phi_{2,3}$ over the bifurcation loci obtained with the technique described in the previous section.

\section{A. Stage 1: Graphical determination of coexisting solutions}

As shown in [1]-[3], the constant inter-stage phase shift $\phi_{1,2}=\phi_{2,3}=\phi$ is varied by detuning the two outermost oscillators. For each pair of tuning voltages $\Delta \eta_{10}, \Delta \eta_{20}$, the constant phase-shift solution coexists with other undesired solutions [11]. To obtain efficiently all the coexisting solutions, a double sweep is performed in $\phi_{1,2}, \phi_{2,3}$ solving for $\Delta \eta_{1}, \Delta \eta_{2}$ at each sweep point. Then, two contours are plotted in the plane $\phi_{1,2}, \phi_{2,3}$. One of them corresponds to $\Delta \eta_{1}=\Delta \eta_{10}$ and the other corresponds to $\Delta \eta_{2}=\Delta \eta_{20}$. The contour $C\left(\Delta \eta_{10}\right)$ is composed by all the phase shift pairs $\phi_{1,2}, \phi_{2,3}$ obtained with $\Delta \eta_{10}$, regardless of the value of $\Delta \eta_{2}$. In a similar manner, the contour $\mathrm{C}\left(\Delta \eta_{20}\right)$ is composed by all the phase shift pairs $\phi_{1,2}, \phi_{2,3}$ obtained with $\Delta \eta_{20}$, regardless of the value of $\Delta \eta_{1}$. The intersection points of these two contours $C\left(\Delta \eta_{10}\right) \cap C\left(\Delta \eta_{20}\right)$ provide the phase values of all the solutions coexisting for $\Delta \eta_{10}, \Delta \eta_{20}$.

As an example, the above method has been applied to obtain all the solutions coexisting with the one having constant interstage phase shift $\phi_{1,2}=\phi_{2,3}=\phi=0^{\circ}$, in the coupled system with the electrical length $\theta_{1}=\theta_{2}=\theta=345^{\circ}$. The corresponding tuning parameter values are
$\Delta \eta_{10}=0.215 \mathrm{~V}, \Delta \eta_{20}=0.215 \mathrm{~V}$. The resulting contours $\mathrm{C}\left(\Delta \eta_{10}\right), C\left(\Delta \eta_{20}\right)$ are shown in Fig. 11. As can be seen, there are four intersections labeled $S_{1}$ to $S_{4}$, corresponding to the four coexisting solutions. Solution $\mathrm{S}_{1}$ corresponds to the constant phase shift $\phi=\phi_{1,2}=\phi_{2,3}=0^{\circ}$.

Now, variations of the constant phase shift $\phi$ between $-180^{\circ}$ to $180^{\circ}$ will be considered. All the solutions that coexist with the ones fulfilling $\phi_{1,2}=\phi_{2,3}=\phi_{\mathrm{c}}$, where $\phi_{\mathrm{c}}$ goes from $-180^{\circ}$ to $180^{\circ}$ and the subindex "c" indicates "curve", can be obtained in an automatic manner. This is done by detecting for each $\phi_{\mathrm{c}}$ value the intersection points between the two contours $C\left(\Delta \eta_{1 \mathrm{cc}}\right), C\left(\Delta \eta_{2 \mathrm{c}}\right)$. This procedure has been applied to the system with electrical length $\theta_{12}=\theta_{23}=\theta=345^{\circ}$. In Fig. 12, the phase-shift values $\phi_{1,2}, \phi_{2,3}$ of the solutions that coexist with the constant phase-shift one $\phi_{1,2}=\phi_{2,3}=\phi_{\mathrm{c}}$ are plotted versus $\phi_{\mathrm{c}}$. In Fig. 12(a), the extended phase interval $-360^{\circ} \leq \phi_{\mathrm{c}} \leq 360^{\circ}$ has been considered, for better interpretation of the solution-curve patterns. A detailed representation in the $-180^{\circ} \leq \phi_{\mathrm{c}} \leq 180^{\circ}$ interval is shown in Fig. 12(b). As can be seen, there are four turning points in the curves corresponding to $\phi_{1,2}$ and $\phi_{2,3}$, marked as $T_{1}$ to $T_{4}$. For $\phi_{c}$ values in the intervals $\left[\phi_{c}\left(T_{1}\right), \phi_{c}\left(T_{2}\right)\right]$ and $\left[\phi_{c}\left(T_{3}\right), \phi_{c}\left(T_{4}\right)\right]$ there are two co-existing solutions and outside this interval there are four.

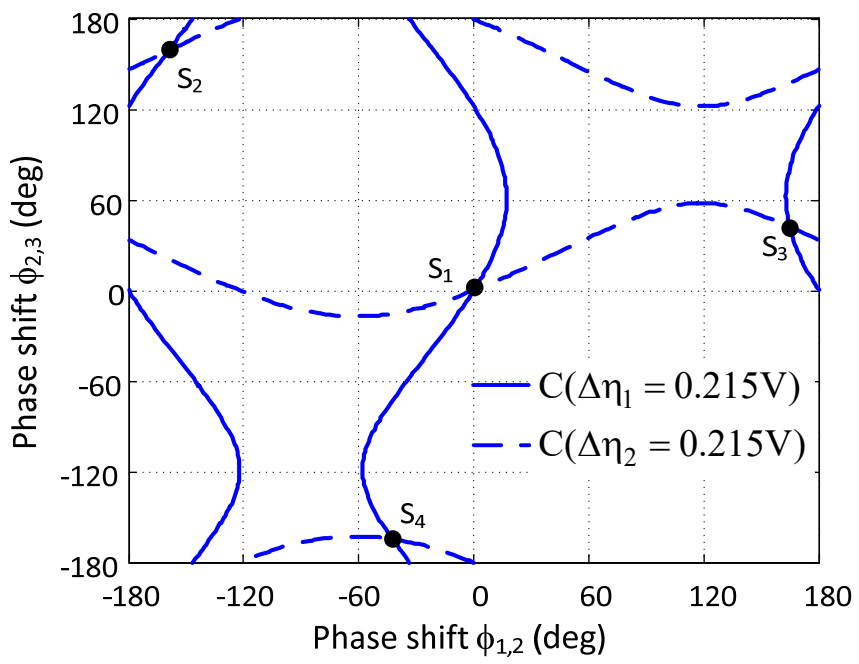

Fig. 11. Graphical determination of the steady-state solutions coexisting with the one with constant inter-stage phase shift $\phi=0^{\circ}$ for the optimal coupling-line electrical length $\theta=345^{\circ}$. The constantparameter contours $C\left(\Delta \eta_{10}\right), C\left(\Delta \eta_{20}\right)$ are traced on the plane defined by the two inter-stage phase shifts $\phi_{1,2}, \phi_{2,3}$. The coexisting solutions correspond to the four contour intersections $\mathrm{S}_{1}$ to $\mathrm{S}_{4}$.

The turning point is a usual class of D-type bifurcation, in which the real pole of zero value gives rise to an infinite slope of the solution curve versus the parameter. This geometrical condition is directly related to the singularity of the Jacobian matrix in (8). Due to this singularity, each turning point bifurcation will give rise to an infinite slope of the steady-state variables $\Delta \mathrm{V}_{1}, \Delta \mathrm{V}_{2}, \Delta \mathrm{V}_{3}, \Delta \omega, \phi_{1,2}, \phi_{2,3}$ versus the tuning 
parameter $\Delta \eta_{1}$ and also versus the parameter $\Delta \eta_{2}$. Using equation (1), it would be possible to obtain a continuous function $\phi_{\mathrm{c}}\left(\Delta \eta_{1}, \Delta \eta_{2}\right)$. This is why, as shown in Fig. 9(b), the solution curves exhibit infinite slope versus $\phi_{\mathrm{c}}$ at each turning point bifurcation.

In Fig. 13, the optimum electrical length $\theta=363.5^{\circ}$, fulfilling the two conditions $\sin \alpha_{\mathrm{v}, \mathrm{nb}}=0$ and $\cos \alpha_{\mathrm{v}, \mathrm{nb}}<0$, has been considered. In this case, the diagram in Fig. 12(b) degenerates into the one in Fig. 13. The curves formerly exhibiting turning points become overlapped with the unitslope straight line.

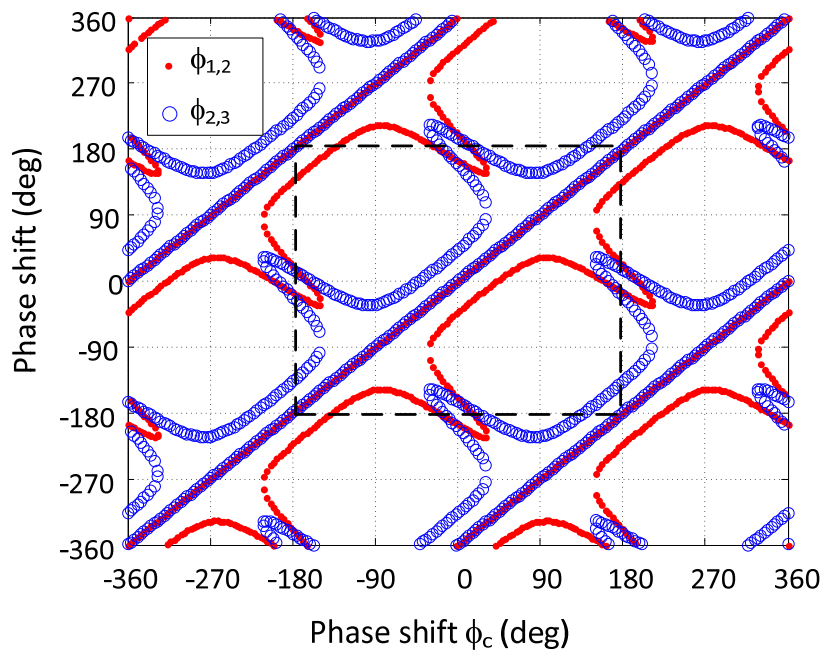

(a)

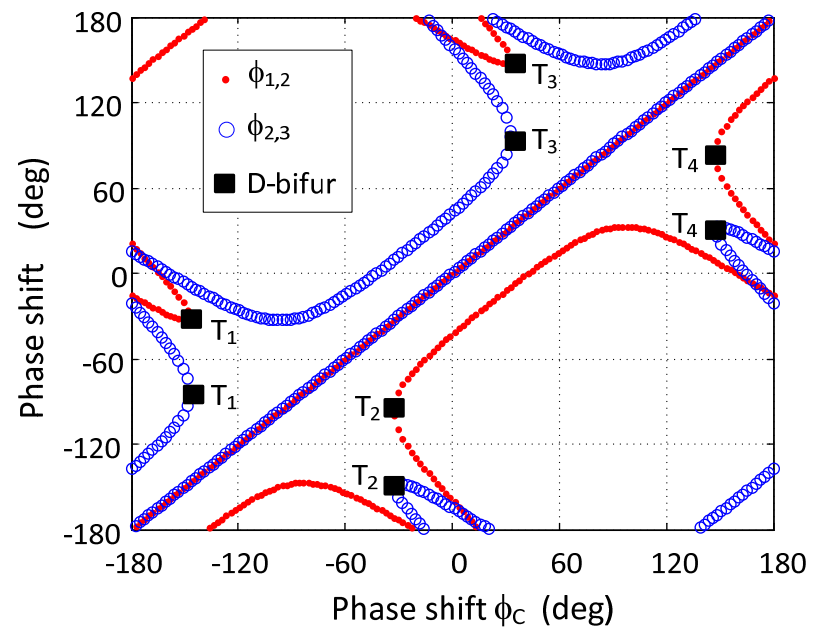

(b)

Fig. 12. Electrical length $\theta=345^{\circ}$. Solution curves coexisting with the constant phase-shift one $\phi_{\mathrm{c}}=\phi_{1,2}=\phi_{2,3}$, represented by tracing their corresponding phase values $\phi_{1,2}, \phi_{2,3} \quad$ versus the intended constant phase shift $\phi_{\mathrm{c}}$. (a) Phase-shift interval $-360^{\circ} \leq \phi_{\mathrm{c}} \leq 360^{\circ}$. (b) Zoom to the interval $-180^{\circ} \leq \phi_{\mathrm{c}} \leq 180^{\circ}$. Turning points are shown with black squares.

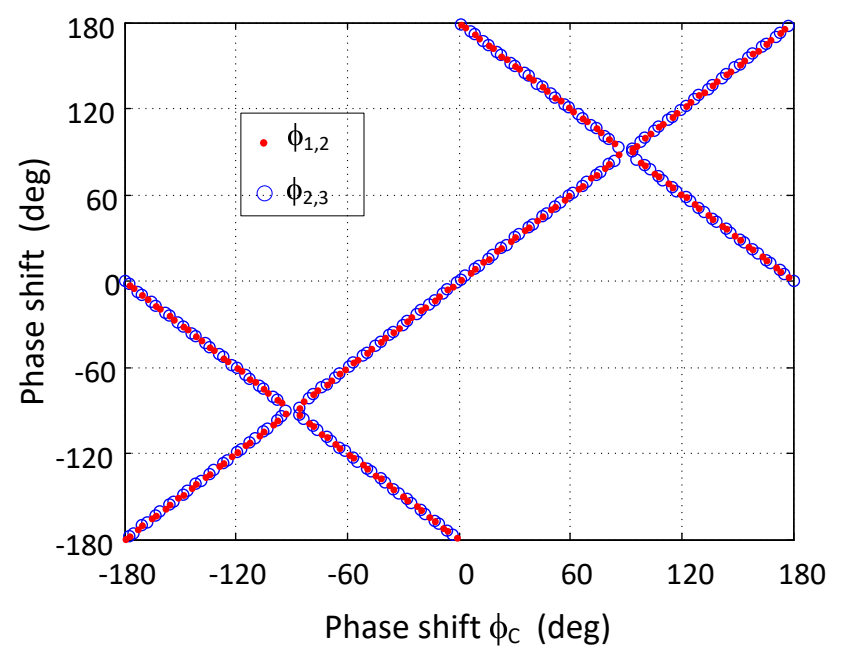

Fig. 13. Solution curves coexisting with the constant phase-shift one $\phi_{\mathrm{c}}=\phi_{1,2}=\phi_{2,3}$ for the optimum electrical length $\theta=363.5^{\circ}$, represented in the interval $-180^{\circ} \leq \phi_{\mathrm{c}} \leq 180^{\circ}$.

\section{B. Stage 2: Stability analysis}

The stability of the different sections of all the solutions curves coexisting with the constant-phase-shift one $\left(\phi_{\mathrm{c}}=\phi_{1,2}=\phi_{2,3}\right)$ can be graphically determined by plotting these curves on the bifurcation loci. This has been done for the solution curves obtained in the analyses of Fig. 12 and Fig. 13, and corresponding to the electrical lengths $\theta=345^{\circ}$ and $\theta=363.5^{\circ}$ (optimal), respectively.

In Fig. 14(a), corresponding to $\theta=345^{\circ}$, all the solution curves $\phi_{1,2} \neq \phi_{2,3} \quad$ coexisting with $\phi_{\mathrm{c}}=\phi_{1,2}=\phi_{2,3}$ are superimposed with dotted lines on the loci of Fig. 4(b). As already known, the constant phase-shift solution (unit-slope straight line) lies on the stable region in the phase interval $\left[-90^{\circ}, 90^{\circ}\right]$. As shown in Fig. 14(a), the additional solution curves traced in Fig. 12 enter the stable region in the section comprised between the turning points $T_{2}$ and $T_{3}$. However, this section is obtained for tuning voltages $\Delta \eta_{1}, \Delta \eta_{2}$ different from those required to vary the constant inter-stage phase shift. The curves obtained by composing the tuning voltages $\Delta \eta_{1}, \Delta \eta_{2}$ through the stable constant phase-shift progression $\left(-90^{\circ}, 90^{\circ}\right)$ and through the section $\mathrm{T}_{2}-\mathrm{T}_{3}$ are shown in Fig. 14(b). As can be seen, they do not intersect. Therefore, there is no coexistence of the stable constant phase-shift solution with other stable solutions having different values of inter-stage phase shifts $\phi_{1,2} \neq \phi_{2,3}$. The representation carried out in Fig. 14 provides the full stability properties (number and nature of the possible unstable poles) of each particular solution. At the turning points $T_{1}$ to $T_{4}$ of the curves of Fig. 12, the associated curves on the plane $\phi_{1,2}, \phi_{2,3}$ in Fig. 14 cross the D-type bifurcation loci. This is in full agreement with the turning points of the solution curves obtained in Fig. 12 and constitutes an independent validation of the new method. 


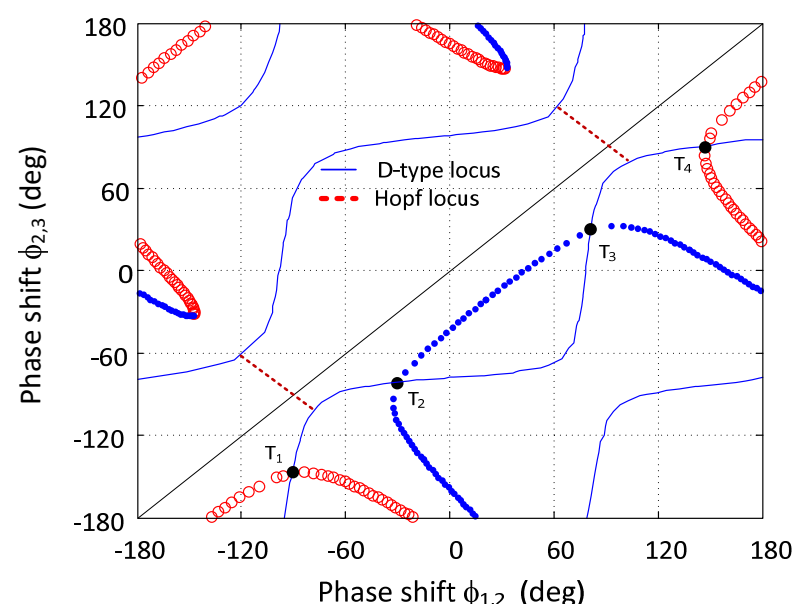

(a)

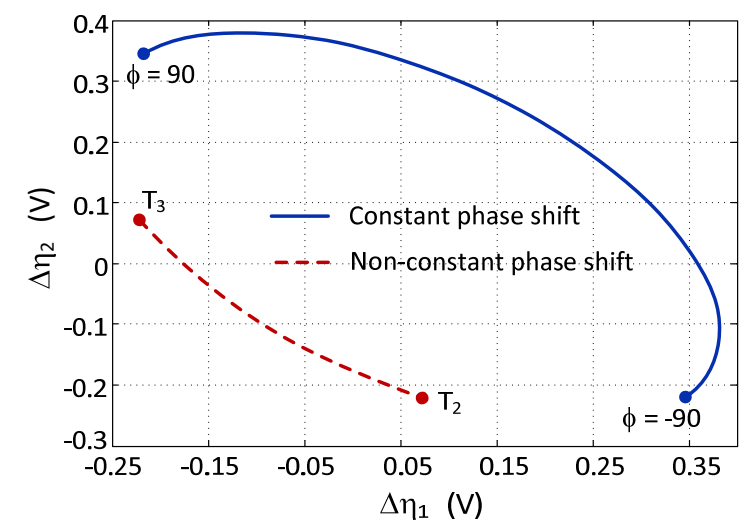

(b)

Fig. 14. Electrical length $\theta=345^{\circ}$. Stability analysis of all the solution curves coexisting with the constant phase-shift one $\phi_{\mathrm{c}}=\phi_{1,2}=\phi_{2,3}$. (a) The stability is analyzed by superimposing these solution curves (dotted line) on the bifurcation loci, traced in the plane defined by the two inter-stage phase shifts $\phi_{1,2}, \phi_{2,3}$, as in Fig. 4(b). At the turning points $T_{1}$ to $T_{4}$ the solution curves cross the $D_{-}$ type bifurcation loci. (b) Curves obtained by composing the tuning voltages $\Delta \eta_{1}, \Delta \eta_{2}$ through the stable constant phase-shift progression $\left(-90^{\circ}, 90^{\circ}\right)$ and through the section $\mathrm{T}_{2}-\mathrm{T}_{3}$. They do not intersect.

In Fig. 15, corresponding to the optimal electrical length $\theta=363.5^{\circ}$, the solution curves coexisting with $\phi_{\mathrm{c}}=\phi_{1,2}=\phi_{2,3} \quad$ (previously obtained in Fig. 13) are superimposed (with dotted line) on the bifurcation loci corresponding to this electrical length value [Fig. 7(b)]. Also in this case, the additional solution curves remain in the unstable regions for all the parameter values. Therefore, there is no coexistence of the stable constant phase-shift solutions with other stable solutions.

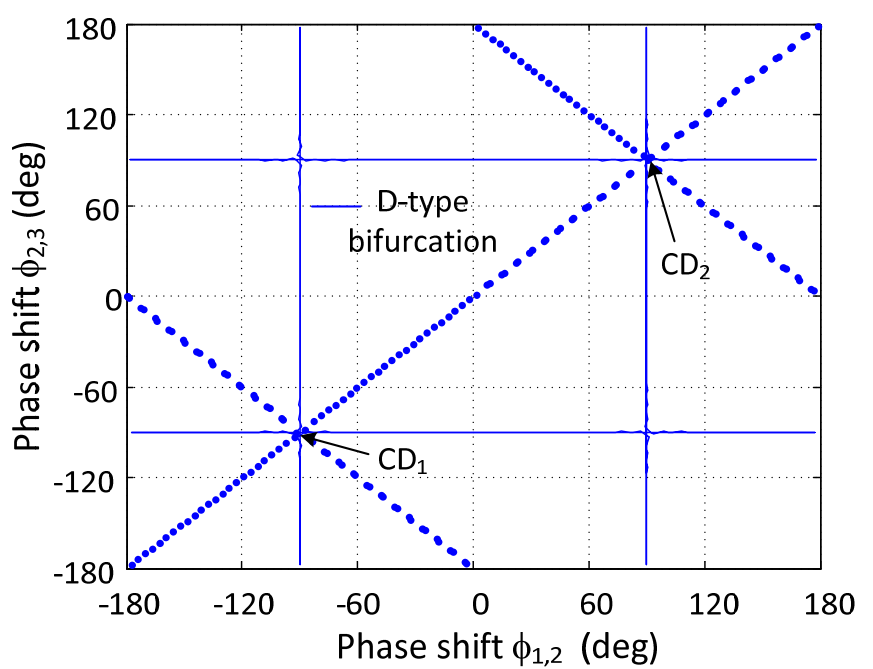

Fig. 15. Electrical length $\theta=363.5^{\circ}$ (optimal). Stability analysis of all the solution curves coexisting with the constant phase shift one $\phi_{\mathrm{c}}=\phi_{1,2}=\phi_{2,3}$. The stability is analyzed by superimposing these solution curves (dotted line) on the bifurcation loci, traced in the plane defined by the two inter-stage phase shifts $\phi_{1,2}, \phi_{2,3}$, as in Fig.

7(b). Operation near the co-dimension two bifurcations is marked as $\mathrm{CD}_{1}$ and $\mathrm{CD}_{2}$.

\section{CONCLUSION}

A new methodology for the stability analysis of coupledoscillator systems, considering all possible values of interstage phase shift, has been presented. It is based on the determination of the bifurcation loci in the space defined by the inter-stage phase shifts, where these solutions are univocally defined. The method is computationally very efficient, as it does not require an initial evaluation of the steady-state solution. It enables an efficient detection of both ordinary and co-dimension two bifurcations and provides a detailed description of the system global stability properties, presented in this work for the first time to our knowledge. This thorough bifurcation analysis allows the comprehension of stability changes commonly observed during the system tuning. The bifurcation loci facilitate the evaluation and increase of the stability margins. The new methodology has been applied to a practical coupled-oscillator system at $3.85 \mathrm{GHz}$, investigating the impact of the length of the transmission line in the coupling networks on the bifurcation loci. The analysis results have been validated with independent simulations and with experimental measurements.

\section{REFERENCES}

[1] R. A. York, "Nonlinear analysis of phase relationships in quasi-optical oscillator arrays," IEEE Trans. Microw. Theory Tech., vol. 41, no. 10, pp. 1799 - 1809, Oct. 1993.

[2] R.A. York, P. Liao and J.J. Lynch, "Oscillator array dynamics with broadband N-port coupling networks," IEEE Trans. Microw. Theory Tech., vol. 42, no. 11, pp. 2040-2042, Nov. 1994.

[3] T. Heath, "Beam steering of nonlinear oscillator arrays through manipulation of coupling phases," IEEE Transactions on Antennas and Propagation, vol. 52, no. 7, pp. 1833-1842, Jul. 2004.

[4] S. Nogi, J. Lin, and T. Itoh, "Mode analysis and stabilization of a spatial power combining array with strongly coupled oscillators," IEEE 
Transactions on Microwave Theory and Techniques, vol. 41, no. 10, pp. 1827-1837, Oct. 1993.

[5] M.R. Kuhn, and E.M. Biebl, "Power combining by means of harmonic injection locking," IEEE MTT-S International Microwave Symposium Digest, Fort Worth , TX, June 2004, vol.1, pp. 91-94.

[6] T. Heath, "Simultaneous Beam Steering and Null Formation With Coupled, Nonlinear Oscillator Arrays," IEEE Transactions on Antennas and Propagation, vol. 53, no. 6, pp. 2031-2035, June 2005.

[7] T. Ikuma, A. A. Beex, J. R. Zeidler, B. K. Meadows, "Adaptive Interference Mitigation with a Coupled Nonlinear Oscillator Array Beamformer," 2006 IEEE Aerospace Conference, Big Sky MT, pp 1-13, 4-11 March 2006.

[8] H. Steyskal, "Simple Method for Pattern Nulling by Phase Perturbation," IEEE Transactions on Antennas and Propagation, vol. AP-31, no. 1, pp.163-166, Jan. 1983.

[9] A. D. Khzmalyan, A. S. Kondratiev, "The Phase-Only Shaping and Adaptive Nulling of an Amplitude Pattern," IEEE Transactions on Antennas and Propagation, vol. 51, no. 2, pp. 264-272, Feb. 2003.

[10] A. Georgiadis, A. Collado, A. Suarez, "New Techniques for the Analysis and Design of Coupled-Oscillator Systems", IEEE Trans. Microwave Theory and Techniques, vol. 54, no. 11, pp. 3864-3877 Nov. 2006.

[11] A. Collado, A. Suarez, S. Sancho, "Semi-analytical formulation for the stability analysis of coexisting solutions in coupled-oscillator systems," IEEE MTT-S International Microwave Symposium Digest, Honolulu, HI, Jun. 2007, pp. 973 - 976.

[12] A. Suárez, Analysis and Design of Autonomous Microwave Circuits, Hoboken, New Jersey, Wiley - IEEE Press, 2009.

[13] A. Georgiadis, A. Collado. A. Suárez. "Pattern Nulling in Coupled Oscillator Antenna Arrays," IEEE Trans. Antennas and Propagation, vol. 55, no.5, pp. 1267-1274, May, 2007.

[14] A. Suárez, F. Ramírez, S. Sancho, "Stability and Noise Analysis of Coupled-Oscillator Systems," IEEE Trans. Microw. Theory Tech., vol. 59, no. 4, pp. 1032-1046, Apr., 2011.

[15] A. Suárez, S. Sancho, F. Ramírez. "General Formulation for the Analysis of Injection-Locked Coupled-Oscillator Systems," IEEE Trans. Microw. Theory Techn., vol. 61, no. 12, pp. 4730-4744, Dec., 2013.

[16] A. Suárez, S. Sancho, F. Ramírez, "Stochastic characterization of the phase noise spectrum of coupled-oscillator circuits", IEEE MTT-S Int. Microwave Symp., Anaheim, CA, USA, Jun., 2010.

[17] A. Suárez, S. Sancho, F. Ramírez, "Explicit formulation for injectionlocked coupled-oscillator systems," IEEE MTT-S Int. Microwave Symp., Seattle, WA, USA, Jun., 2013.

[18] J. Guckenheimer and P. Holmes, Nonlinear Oscillations, Dynamic Systems, and Bifurcations of Vector Fields. New York: SpringerVerlag, 1983.

[19] S. Wiggins, Introduction to Applied Nonlinear Dynamical Systems and Chaos. New York: Springer-Verlag, 1990.

[20] F. Ramírez, E. de Cos, A. Suárez, "Nonlinear analysis tools for the optimized design of harmonic-injection frequency dividers", IEEE Trans. Microw. Theory Tech., vol. 51, no. 6, pp. 1752-1762, Jun. 2003.

[21] V. Rizzoli and A. Neri, "State of the art and present trends in nonlinear microwave CAD techniques," IEEE Trans. Microw. Theory Tech., vol. 36, no. 2, pp. 343-365, Feb. 1988.

[22] LAPACK Users' Guide (3rd Ed.) [Online]. Available: http://www.netlib.org/lapack/

[23] G. Iooss and D. D. Joseph, Elementary Stability and Bifurcation Theory. New York: Springer-Verlag, 1997.

[24] J. Marsden and M. McCracken, Hopf Bifurcation and Its Applications. New York: Springer-Verlag, 1976.

[25] J. Carr, Application of the Centre Manifold Theory, volume 35 of Applied Mathematical Sciences. New York: Springer-Verlag, 1981

[26] J. de Cos, A. Suárez, and F. Ramírez, "Analysis of oscillation modes in free-running ring oscillators," IEEE Trans. Microw. Theory Tech., vol. 60, no. 10, pp. 3137-3150, Oct. 2012.

[27] K. Kurokawa, "Some basic characteristics of broadband negative resistance oscillators," The Bell System Technical Journal, vol. 48, pp. 1937-1955, July-August. 1969.

[28] R. Adler, "A study of locking phenomena in oscillators," Proceedings of the IEEE, vol. 61, no.10, pp. 1380 - 1385, Oct. 1973.
Almudena Suárez (M'96-SM'01-F'12) was born in Santander, Spain. She received the Electronic Physics and Ph.D. degrees from the University of Cantabria, Santander, Spain, in 1987 and 1992, respectively, and the Ph.D. degree in electronics from the University of Limoges, Limoges, France, in 1993. She is currently a Full Professor with the Communications Engineering Department, University of Cantabria. She co-authored Stability Analysis of Nonlinear Microwave Circuits (Artech House, 2003) and authored Analysis and Design of Autonomous Microwave Circuits (IEEE-Wiley, 2009). Prof. Suárez is a member of the Technical Committees of the IEEE International Microwave Symposium (IMS), the European Microwave Conference. She was an IEEE Distinguished Microwave Lecturer from 2006 to 2008, with the talk "Stability analysis and stabilization of power amplifiers." She is the editor-in-chief of International Journal of Microwave and Wireless Technologies, from Cambridge Journals. She is a member of the "Board of Directors" of the European Microwave Association. She was the coordinator of the Communications and Electronic Technology Area for the Spanish National Evaluation and Foresight Agency (ANEP) between 2009 and 2013.

Franco Ramírez was born in Potosí, Bolivia. He obtained a degree in electronic systems engineering degree from the Military School of Engineering (EMI) in La Paz, Bolivia, in 2000 and the Ph.D. degree in communications engineering from the University of Cantabria, Santander, Spain in 2005. From 1999 to 2000 he worked for Ericsson de Bolivia Telecomunicaciones, where he was involved in several projects related with GSM and TDMA technologies. At present he is a Research Associate, under the "Ramón y Cajal" Programme of the Spanish Ministry of Science and Innovation, at the Communications Engineering Department of the University of Cantabria. His research interests include phase noise, stability and the development of nonlinear techniques for the analysis and design of autonomous microwave circuits.

Sergio Sancho was born in Santurce, Spain, in 1973. In 1997 received the degree in Physics from Basque Country University. In 1998 he joined the Communications Engineering Department of the University of Cantabria, Spain, where he received the Ph.D. degree in Electronic Engineering in February 2002. At present, he works at the University of Cantabria, as an Associate Professor of its Communications Engineering Department. His research interests include the nonlinear analysis of microwave autonomous circuits and frequency synthesizers, including stochastic and phase-noise analysis.

Juan-Mari Collantes received the Ph.D. degree in electronics from the University of Limoges, France, in 1996. Since February 1996, he has been an Associate Professor with the Electricity and Electronics Department, University of the Basque Country (UPV/EHU), Bilbao, Spain. In 1996 and 1998 he was an Invited Researcher with Agilent Technologies (formerly the Hewlett-Packard Company), Santa Rosa, CA. In 2003, he was with the French Space Agency (CNES), Toulouse, France, where he was involved with power amplifier analysis, simulation, and modeling. His areas of interest include nonlinear analysis and design of microwave circuits, microwave measurement techniques and noise characterization. 